


\title{
Marco TOE para diferenciar la asimilación del ERP en franquicias y empresas familiares mexicanas
}

\author{
Silvia Leticia López Rivas, Jannett Ayup González \\ y Adriana Méndez Wong
}

\section{UNIVERSIDAD AUTÓNOMA DE CIUDAD JUÁREZ \\ PUBLICACIÓN AFILIADA A LA \\ RED IBEROAMERICANA DE ESTUDIOS DEL DESARROLLO}




\title{
UNIVERSIDAD AUTÓNOMA DE CIUDAD JUÁREZ
}

\author{
PUBLICACIÓN AFILIADA A LA \\ RED IBEROAMERICANA DE ESTUDIOS DEL DESARROLLO
}

Universidad Autónoma de Ciudad Juárez 2018-2024

Mtro. Juan Ignacio Camargo Nassar

Rector

Mtro. Daniel Alberto Constandse Cortez

Secretario General

Mtro. Santos Alonso Morales Muñoz Director del Instituto de Ciencias Sociales y Administración Mtro. Jesús Meza Vega

Director General de Comunicación Universitaria

Comité de Coordinación de la Red Iberoamericana de Estudios del Desarrollo 2018-2020

Dra. Paulina Sanhueza Martínez (Universidad de la Frontera, Chile)

Coordinadora General

Dr. Ignacio Rodríguez Rodríguez (Universidad de la Frontera, Chile)

Secretario general

Dra. Myrna Limas Hernández

(Universidad Autónoma de Ciudad Juárez, México)

Vocal de Organización

Dr. Pablo Galaso Reca (Universidad de la República, Uruguay)

Vocal de Organización

Dr. Luis Enrique Gutiérrez Casas

Director y editor de Cuadernos de Trabajo

Estudios Regionales en Economía, Población y Desarrollo

Comité editorial

Sección internacional Dra. Sofía Boza Martínez

(Universidad de Chile, Chile)

Dra. Olga Biosca Artiñano

(Glasgow Caledonian University, Reino Unido)

Dra. Ángeles Sánchez Díez

(Universidad Autónoma de Madrid, España)

Dr. Thomas Fullerton Mankin

(University of Texas at El Paso, Estados Unidos)

Dr. Adrián Rodríguez Miranda

(Universidad de la República, Uruguay)

Dra. Ikuho Kochi

(Kanazawa University, Japón)

Dr. Pablo Galaso Reca

(Universidad de la República, Uruguay)

Sección local

(Universidad Autónoma de Ciudad Juárez)

Dra. Myrna Limas Hernández

Dra. Rosa María García Almada

Dr. Raúl Alberto Ponce Rodríguez

Dr. Isaac Leobardo Sánchez Juárez

Dr. Héctor Alonso Barajas Bustillos

Dr. Juan Carlos Medina Guirado

Mtra. María Del Socorro Velázquez Vargas

Diseño de cubierta

Abigail Bautista
Estudios Regionales en Economía, Población

y Desarrollo. Cuadernos de Trabajo de la UACJ

ISSN 2007-3739

Número 65. Septiembre - Octubre 2021

Marco TOE para diferenciar la asimilación del ERP en

franquicias y empresas familiares mexicanas

Silvia Leticia López Rivas, Jannett Ayup González

y Adriana Méndez Wong

Universidad Autónoma de Ciudad Juárez

Estudios Regionales en Economía, Población y Desarrollo.

Cuadernos de Trabajo de la UACJ

Año 11, No. 65 septiembre - octubre 2021, es una publicación bimestral editada por la Universidad Autónoma de Ciudad Juárez a través del Cuerpo Académico de Estudios Regionales en Economía, Población y Desarrollo del Instituto de Ciencias Sociales y Administración. Redacción: Avenida Universidad y H. Colegio Militar, Zona Chamizal s/n., C.P. 32300, Ciudad Juárez, Chihuahua, México.

Teléfonos: (656) 688-38-00, ext.3792. Correo electrónico: igtz@uacj.mx. Editor responsable: Luis Enrique Gutiérrez Casas. Reserva de derechos al uso exclusivo: edición impresa, ISSN 2007-3739., edición digital, No. de reserva 04-2019-050218151500. Impresa por Studio Los Dorados, calle Del Campanario, número 820-2, Santa Cecilia, C.P. 32350, Cd. Juárez, Chihuahua. Distribuidor: Subdirección de Gestión de Proyecto y Marketing Editorial. Ave. Plutarco Elías Calles 1210, Foviste Chamizal, C.P. 32310, Ciudad Juárez, Chihuahua. Este número se terminó de imprimir el 15 de agosto 2021 con un tiraje de 120 ejempares.

Los ensayos publicados son responsabilidad exclusiva de sus autores.

Se autoriza la reproducción total o parcial bajo condición de citar la fuente.

Registrada en:

UAC] 侧 Revistas Electrónicas

Publicación afiliada a la Red Iberoamericana de Estudios del Desarrollo

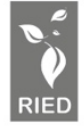

Universidad Autónoma de Ciudad Juárez

Ave Plutarco Elías Calles 1210

Foviste Chamizal, C.P. 32310

Ciudad Juárez, Chihuahua, México

www.uacj.mx

(C) Universidad Autónoma de Ciudad Juárez 
Estudios Regionales en Economía, Población y Desarrollo. Cuadernos de Trabajo, Universidad Autónoma de Ciudad Juárez, publicación afiliada a la Red Iberoamericana de Estudios del Desarrollo, número 65, septiembre-octubre de 2021, ISSN 2007-3739, pp. 3-36 México.

\title{
Marco TOE para diferenciar la asimilación del ERP en franquicias y empresas familiares mexicanas
}

\author{
Silvia Leticia López Rivas *, Jannett Ayup González ** \\ y Adriana Méndez Wong ***
}

\begin{abstract}
Resumen
La importancia de las franquicias y empresas familiares en la econom ía es indiscutible; sin embargo, hay pocos estudios enfocados a valorar la asimilación de sistemas de planeación empresarial (ERP, por sus siglas en inglés), en lo particular, en el sector restaurantero. Esta investigación busca determinar si la relació $n$ entre el uso del ERP y el desempeño económico es distinto entre las franquicias y las empresas familiares. En el análisis se utiliza la información de una muestra de 70 franquicias y 102 empresas familiares mexicanas. Este estudio resalta diferencias significativas entre ambos tipos de empresas. Cabe mencionar que, independientemente del tipo de negocio de que se trate, lo que arroja un mejor resultado en ambas muestras es la presión competitiva del uso del ERP por sus competidores y el papel que juega la transferencia de conocimiento.
\end{abstract}

Palabras clave: Modelo TOE, ERP, franquicias, empresas familiares, PLS-SEM.

\section{TOE framework to differentiate the assimilation of ERP in franchises and Mexican family-owned businesses}

\begin{abstract}
The importance of franchises and family businesses in the economy is unquestionable. However, there are a few studies focused on the assimilation of enterprise resource planning system (ERP) in these types of businesses, especially, food and restaurant service sector. This article aims to determine if the relationship between the assimilation of the ERP and the performance for franchises and family-owned businesses have differential effects. The analysis use sample information of 70 franchises and 102 Mexican family-owned businesses. This study shows that the most important differences between both businesses' classifications are present in the ERP effect on financial performance for family businesses and non-financial performance for franchises. Regardless the type of business, what drives this assimilation for a better result is the competitive pressure of the use of ERP by its competitors and the role that knowledge transfer plays.
\end{abstract}

Keywords: TOE Model, ERP, franchise, family firms, PLS-SEM. JEL: D22, M10, M21.

- Recibido en: Febrero de 2021. - Aprobado en: Junio de 2021.

\footnotetext{
* Adscrita a la Facultad de Comercio y Administración de la Universidad Autónoma de Tamaulipas (México). Correo electrónico:sllopez@docentes.uat.edu.mx. ORCID:0000-0001-6714-5667.

** Adscrita a la Facultad de Comercio y Administración de la Universidad Autónoma de Tamaulipas (México). Correo electrónico: yanethay@uat.edu.mx. ORCID:0000-0003-0433-5679.

***Adscrita a la Facultad de Mercadotecnia de la Universidad Autónoma de Coahuila (México). Correo electrónico: adrianamendezwong@uadec.edu.mx. ORCID:0000-0003-3935-1265.
} 


\section{$\rightarrow$ 1. Introducción.}

Algunas investigaciones sugieren que los factores de las funciones organizacionales y gerenciales son resultado de la efectividad, el éxito y el desempeño del proceso de los sistemas de información en la gestión de empresa (Garg y Chauhan, 2015). Tal es el caso de las aplicaciones informáticas de planificación de recursos empresariales (ERP) que son paquetes de sistemas de información, que consisten en bases de datos comunes, que ayudan a la gestión de las empresas, grandes y medianas, a través de la recopilación y el procesamiento de los datos para el análisis de la información (OciepaKubicka, 2017). Estos sistemas son conocidos como Enterprise Resource Planning (ERP).

Un sistema del ERP favorece las condiciones para que la empresa sea rentable y competitiva; sin embargo, las empresas continúan con diversos retos en el proceso, tales como la actitud de los integrantes de la organización hacia los cambios y las formas distintas de trabajo, tanto para implementar el sistema como en su asimilación, a pesar de los esfuerzos de las investigaciones y los avances tecnológicos (Phaphoom, Saelee, Somjaitaweeporn, Yuenyong y Qu, 2018). La inversión en sistemas de gestión empresarial, además de considerarse obligatorio para la supervivencia de la empresa, es un factor que aumenta la eficiencia de las actividades administrativas y operacionales, en la actual transformación digital empresarial, con la finalidad de mejorar el rendimiento de tales actividades (Peppard, 2016) pero no siempre implican eficacia organizativa, en virtud de la falta de habilidades directivas (Bruhn, Karlan y Schoar, 2012), así como una deficiente visión estratégica (Mendoza, Mendoza y Guzmán, 2018), entre otras.

Es importante mencionar que, pocos estudios han realizado investigaciones sobre el valor del ERP en la etapa de asimilación en los países en desarrollo (Ghobakhloo, Azar, y Tang, 2018), donde el valor del sistema se refiere particularmente al impacto sobre el desempeño de la empresa $(\mathrm{Xu}, \mathrm{Ou}$, y Fan, 2017). Huang y Yasuda (2016) señalan que se han iniciado las investigaciones en la etapa de asimilación del conocimiento creado desde el ERP, pero en industrias muy específicas, por lo que no se puede generalizar. Si bien las grandes empresas tienen más propensión a adoptar aplicaciones digitales (Kannabiran y Dharmalingam, 2012), las pequeñas empresas en su afán de lograr competitividad, son llevadas por necesidad a requerir de los sistemas ERP (Maguire, Koh y Magrys, 2007), careciendo en ocasiones, por su naturaleza, de los recursos adecuados para adoptar un software grande y complejo (Awa y Ojiabo, 2016). Este estudio se enfoca en pequeños y medianos negocios para entender las condiciones en que se ha dado la asimilación del conocimiento al implementar sistemas de gestión empresarial apoyados por la tecnología, concretamente, la industria de servicios alimentarios con formato de negocio de franquicia o empresa familiar. La franquicia es una empresa de naturaleza híbrida por su propiedad y gestión, lo cual facilita su crecimiento y expansión y, 
pudiendo ser una empresa familiar, aquella donde las decisiones importantes se concretan en la familia propietaria. Los negocios adheridos a una red de franquicias se distinguen de las no franquicias por ser una organización apoyada en su operación y comercialización en manuales y procedimientos estandarizados. Los negocios integrados en una red de franquicias tienen mayor participación en el sector servicios, y en éste a su vez, las actividades primordiales se dan en alimentos, bebidas y restaurantes, incluidos los alojamientos de tamaño intermedio.

La razón principal para concentrar el estudio en el sector restaurantero es porque en particular, en el estado de Tamaulipas contribuye al PIB nacional con 1.8\% (INEGI, 2018) con la actividad terciaria 72 que corresponde a Servicios de alojamiento temporal y de preparación de alimentos y bebidas, donde está comprendido dicho sector restaurantero. Tamaulipas presenta una oferta restaurantera importante, diversificada y atractiva, distribuida en puntos estratégicos de cada uno de los municipios considerados, otorgando a la población una amplia variedad de empleos en este sector, que van desde niveles operativos básicos, hasta niveles gerenciales. Además, cabe mencionar que, de acuerdo a datos referidos al cuarto trimestre INEGI (2019), dicho estado tiene una población económicamente activa de 1,719,792, de los cuales hay una población ocupada en el sector terciario de 1,024,585 y, de esta población, trabajan en restaurantes y servicios de alojamiento, cerca del 13\% es decir 133,557 habitantes, motivo también importante para destacar el estudio en este sector restaurantero.

En otras líneas, los estudios relacionados con la medida en que los sistemas de planificación empresarial favorecen el éxito organizacional, por ejemplo, el de Baykasoğlu y Gölcük (2017) han encontrado que aumentan las capacidades de conocimientos, aún con distinta estructura organizacional y de gestión si son utilizados eficazmente dada la asimilación de los sistemas de control de gestión empresariales que hubiesen adoptado y el contexto en que se desenvuelven. Para que dicho sistema de gestión empresarial tenga éxito, se requiere que la organización sea capaz de difundir conocimientos sobre el uso y los beneficios de los sistemas de información, entre sus empleados (Cahyadi, 2019), sin embargo, resulta problemático, percatarse de tales beneficios, ya que no es fácil ni evidente (Maas, Van Fenema y Soeters, 2018). En este estudio se incorporan los elementos del marco TOE (Tecnología-Organización-Ambiente) apoyados en la Teoría de la Visión Basada en los Recursos (RBV), Teoría de la Acción Razonada (TAR) y la Teoría de la Difusión de las Innovaciones (DOI), a fin de lograr el objetivo de la investigación. Con estas consideraciones, se plantea como objetivo de estudio, determinar el efecto de la asimilación de los sistemas de planificación de recursos empresariales en el desempeño en la industria restaurantera en el Noreste de México, distinguiendo las diferencias entre franquicias -como firmas estandarizadas- y empresas familiares -con flexibilidad organizacional. 
El documento se estructura iniciando con la presentación de la literatura revisada destacando la transferencia de conocimiento como factor determinante de la eficacia de gestión, seguido del concepto, investigaciones y teorías utilizadas en la Asimilación del ERP, después el modelo de investigación incluyendo las hipótesis, posteriormente el apartado metodológico, resultados, para finalizar con las conclusiones.

\section{2. Marco teórico y referencial.}

\subsection{Transferencia de conocimiento.}

El valor del conocimiento es crucial en la etapa de asimilación de TI ( tecnologías de información) tal como señalan Fichman y Kemerer (2016), ya que, en esta etapa, las organizaciones obtienen los beneficios de los sistemas de gestión empresarial y logran recuperar la inversión (Abd, Nasr y Geith, 2017). La transferencia de conocimiento se puede explicar por la teoría de Difusión de las Innovaciones (DOI) (Rogers, 1995), la cual explica cómo se lleva a cabo el proceso de comunicación de las innovaciones, destacando la influencia de la socialización de conocimientos y experiencias, sobre las decisiones de los demás. Es importante mencionar en este apartado que, los autores Eze, Awa, Okoye, Emecheta y Anazodo (2013) comentan que algunos modelos y teorías como DOI, TOE, TAM, entre otros, fundamentadas en la Teoría de la Acción Razonada (TAR), tienen sus propias construcciones, pero cada una de ellas aporta a la teoría de adopción subyacente. La Teoría de la Acción Razonada (Fishbein y Azjen, 1975), intenta predecir la conducta humana, describiendo las actitudes individuales, que pueden ser de tipo cognitivo, afectivo y conductual. Y si suponemos que su opinión es correcta, la transferencia de conocimiento estará enmarcada bajo estas dos teorías en nuestra investigación: DOI y TAR.

Reconociendo su importancia, se han realizado diversas investigaciones sobre las estrategias para lograr la transferencia de conocimiento (Cahyadi, 2019), una de las cuales es aquella que se apoya en los sistemas capaces de crear, guardar, compartir y usar el conocimiento existente de la empresa por medio de las TI (Davenport y Prusak, 2000), por lo que se ha propuesto incorporar el conocimiento en la tecnología, como una manera eficaz de transferir conocimiento (Lee, Lee y Lee, 2007). Asumiendo que el conocimiento se origina en la mente de los individuos, se considera una mezcla dinámica de experiencia, valores, información del entorno y conocimiento experimentado, que propicia nuevas experiencias e información, quedándose registradas en documentos, rutinas, procesos, prácticas y normas de la organización (Davenport y Prusak, 2000); el proceso que consiste 
en el intercambio de conocimiento y su aplicación, dado entre la fuente de conocimiento y su receptor, es llamado transferencia de conocimiento (Cahyadi, 2019).

Por otro lado, hay que destacar que, la franquicia es un formato de negocio cuya organización impulsa la transferencia efectiva de conocimiento (Inkpen y Tsang, 2005); en donde el franquiciador, quien concede la gestión de marca, es el generador e intermediario del conocimiento, a través de la conceptualización y documentación del conocimiento, con el objetivo de que el franquiciado, quien opera la comercialización del negocio, pueda emplearlo y difundirlo (Markus, 2001). En el caso de otro tipo de firmas como las empresas familiares, la transferencia de conocimiento muestra un efecto positivo y significativo en la orientación empresarial, lo que a su vez impacta en el rendimiento del negocio (Barroso, Sanguino y Bañegil, 2016).

\subsection{Asimilación del ERP.}

El estudio de Purvis, Sambamurthy y Zmud (2001) sobre asimilación de los sistemas de gestión empresarial se fundamenta en la asimilación de TI, esta asimilación se puede tomar como la medida en la cual una empresa haga uso del sistema en las diferentes actividades de procesos comerciales rutinarios. La literatura relevante de la conectividad y las interfaces interfuncionales en la asimilación del ERP es destacada por Nandi y Vakkayil (2018), el autor encontró en un estudio de caso que el soporte de alta dirección, las percepciones sobre el valor de los sistemas y su motivación para usarlos, impacta directamente en el proceso de asimilación en la organización (Kouki, Poulin y Pellerin, 2014); por otra parte, investigaciones empíricas han mostrado a través de sus resultados, que el apoyo de alta dirección, la innovación del CEO (director ejecutivo), influyen significativamente en el beneficio general del sistemas de gestión mediado por el proceso de asimilación (Govindaraju, Salajar, Chandra y Sudirman, 2015). Gran parte de estos estudios se han centrado en examinar los factores que afectan la asimilación del ERP dentro de la empresa, pero sin considerar el impacto sobre el rendimiento de la organización (Wang, Shih, Jiang y Klein, 2008), y la creación del valor comercial (Er, Zayin y Pamungkas, 2017), lo que ha motivado a realizar esta investigación, de manera integral y considerando los desempeños financieros y no financieros.

\subsection{El modelo TOE.}

Los factores internos en el sistema empresarial que impactan la asimilación de TI, han sido examinados a través de la teoría de la Visión Basada en los Recursos (RBV), la Visión Basada en el Conocimiento (VBC) y la teorización de la estructuración de la tecnología tomando elementos basados en recursos, conocimiento y tecnología que explican los factores organizacionales que intervienen en la asimilación de las TI (Chatterjee, Grewal y Sambamurthy, 2002). Mientras que los 
factores externos que afectan la asimilación de TI han sido estudiados mediante la teoría institucional (Liang, Saraf, Hu y Xue, 2007) para explicar el comportamiento de las reglas de juego que opera la firma y su efecto en la asimilación de las TI, por lo que es preponderante fortalecer un marco integrador que incluya los dos factores: internos y externos. En este sentido, en el estudio de la adopción de tecnología, uso y creación de valor, el marco TOE proporciona una base teórica sobresaliente (Gangwar, Date y Raoot, 2014), en donde el ajuste entre la funcionalidad de la tecnología y los requisitos de tareas es mínimo (Balaid, Abd Rozan y Abdullah, 2017), logrando la aceptación tanto teórica como empírica en el área de sistemas de información (Yoon y George, 2013), por su validez, marcada robustez y su destacada inclusión en el estudio de adopción de tales sistemas (Gangwar et al., 2014). Al respecto Tornatzky y Fleischer (1990), desarrollaron una contribución importante al incorporar en su modelo TOE los aspectos de tecnología, organización y medio ambiente, el cual debe su nombre (por sus siglas en inglés) a los tres aspectos que esta teoría señala tener un efecto en el proceso de implementación organizacional de innovaciones tecnológicas, que incluye, las tecnológicas, las organizacionales y criterios ambientales (Bose y Luo, 2011).

Por su parte, la Visión Basada en los Recursos (RBV), es una teoría que en esta investigación fundamenta el marco TOE, a fin de reforzar de forma concreta el marco teórico orientado a la (1) tecnología, (2) organización y (3) medioambiente, tal como sugieren algunos investigadores, los cuales proponen integrar otras teorías, con la finalidad de identificar factores específicos para los tres contextos, tecnológico, organizacional y ambiental, y poder determinar las relaciones subyacentes entre los factores (Awa, Baridam y Nwibere, 2015). De igual forma, la teoría RBV, se centra en las bases económicas y de gestión (Melville, Kraemer, Gurbaxani, Ew y Kraemer, 2004), ya que, en investigaciones realizadas en sistemas de información (SI), se ha utilizado para demostrar el valor comercial de TI, donde la combinación de recursos concretos define el rendimiento de la empresa (Ruivo, Oliveira y Neto, 2015).

\section{Modelo de investigación y desarrollo de hipótesis.}

\subsection{Desarrollo del modelo de investigación.}

Con base en el marco TOE y atendiendo las características del sistema ERP, se propone un modelo de investigación integral, en donde se considera la asimilación del ERP en función de la tecnología, la organización y el ambiente, en la que la asimilación del ERP impulsaría el desempeño de la organización. El modelo de investigación se presenta en la figura 1, se proponen ocho factores determinados en el contexto TOE, que en investigaciones anteriores se han considerado importantes 
para la asimilación del ERP. Los factores son clasificados en tres aspectos: 1) tecnológico, en donde se considera la ventaja relativa, la compatibilidad y la complejidad; 2) el aspecto organizacional, incluyendo el soporte de alta dirección, ajuste organizacional, compromiso financiero y el factor transferencia de conocimiento, que es el factor diferencial de investigación y por último 3) el aspecto ambiental, considerando la presión competitiva. Además, el modelo presenta el impacto de la asimilación del ERP para la mejora del desempeño de la empresa.

Figura 1

\section{Modelo de investigación}

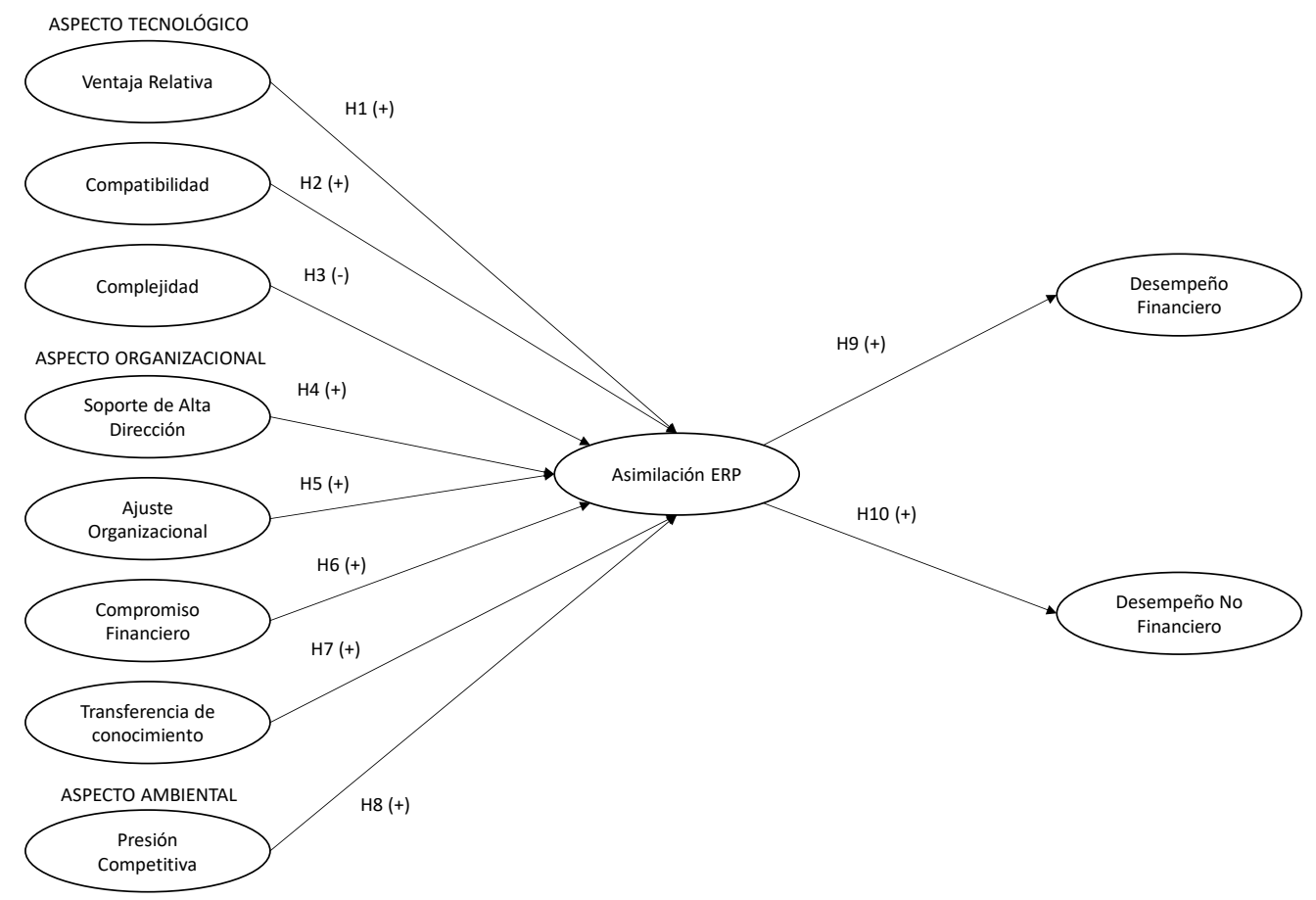

Fuente: elaboración propia basada en Xu, Ou, Fan (2017).

\subsection{Desarrollo de hipótesis.}

\subsubsection{Aspecto tecnológico.}

En el contexto tecnológico se consideran las tecnologías internas y externas que pueden impactar a la organización, y que son relevantes y aplicables para la empresa (Gangwar, Date y Ramaswamy, 2015). Si bien los factores característicos dentro del contexto tecnológico pueden variar entre los diferentes estudios, considerando el entorno ERP, hemos incluido tres factores que son los 
que preponderantemente se han utilizado y reconocido como los propulsores tecnológicos fundamentales en la asimilación ERP: ventaja relativa, compatibilidad y complejidad (AL-Shboul, 2018).

a. Ventaja relativa.

Las innovaciones que tienen una ventaja evidente en la generación de efectividad estratégica y operativa, como aumentar las ventas y disminuir los costos, tienen mayor propensión hacia la adopción de los sistemas de gestión (Greenhalgh, Macfarlane, Bate y Kyriakidou, 2004). La ventaja relativa es "el grado en que una innovación se percibe como mejor que la idea que reemplaza" (Rogers, 1995, p. 15), si los riesgos percibidos son menores que los beneficios otorgados por la innovación, existen más probabilidades de que la empresa la adopte (Ghobakhloo, Arias-Aranda y BenitezAmado (2011). En este sentido, cuando los usuarios perciben la ventaja relativa del sistema ERP como alta, aumentará su uso y con ello la asimilación del ERP, por lo que se dice que la ventaja relativa de una innovación estará relacionada positivamente con su asimilación (Xu et al., 2017). De aquí que, se propone la siguiente hipótesis:

$\mathrm{H}_{1}$ : la ventaja relativa tiene un efecto positivo en la Asimilación del ERP.

b. Compatibilidad.

La compatibilidad es el grado en que coinciden el sistema ERP con las características de TI (Ruivo, Oliveira y Neto, 2014); en un entorno ERP, la asimilación del ERP frecuentemente se manifiesta tanto en la práctica como en los procesos, por lo que la compatibilidad organizacional resulta clave para la asimilación del ERP. Asimismo, la compatibilidad técnica es de suma relevancia, ya que puede integrarse el software existente con el ERP (Bradford y Florin, 2003), por lo que si se presenta una integración entre el sistema ERP y las actividades existentes en la organización, la probabilidad de asimilación será mayor (Delone y Mclean, 1992). Así formulamos la siguiente hipótesis:

$\mathrm{H}_{2}$ : La compatibilidad tiene un efecto positivo en la asimilación del ERP.

c. Complejidad.

A diferencia de otras características de la innovación, la complejidad tiene un efecto negativo en la adopción (Alshamaila, Papagiannidis y Li, 2013). La complejidad se refiere al grado en que una innovación se percibe como complicada de entender, usar y aplicar (Borgman, Bahli, Heier, y Schewski, 2013). Es importante señalar que, la complejidad es una característica propia de los sistemas de cualquier tipo, aunado a que, en la asimilación del ERP se requieren ciertas modificaciones en los departamentos que constituyen la empresa, se presenta una complejidad adicional (Poston y Grabski, 2001), influyendo desde la etapa de la implementación del sistema ERP (Vluggen, 2005) en donde las actitudes de los usuarios del sistema ERP, pueden ser rechazados por 
su complejidad (Chang, Cheung, Cheng y Yeung, 2008) y por consecuencia disminuir la asimilación del sistema de gestión (Xu et al., 2017). Bajo estos argumentos, proponemos la siguiente hipótesis:

$\mathrm{H}_{3}$ : la complejidad tiene un efecto negativo en la asimilación del ERP.

\subsubsection{Aspecto organizacional.}

En el contexto organizativo se identifican las características de la empresa, el estado, su estructura gerencial, la centralización y calidad de los recursos (Awa, Ojiabo y Orokor, 2017; Picoto, Bélanger, y Palma-dos-Reis, 2014) para entender su funcionamiento, eficiencia y eficacia. Basándonos en estudios anteriores y considerando las características específicas del sistema ERP ( Xu et al., 2017; Zhu et al., 2010), clasificamos como los factores más relevantes que pueden influir en la asimilación del ERP dado, el soporte de alta dirección, el ajuste organizacional, el compromiso financiero y la transferencia de conocimiento. Es importante destacar, que la transferencia de conocimiento es un factor distintivo en nuestra investigación, para lo cual nos apoyamos en los autores Awa et al. (2017), con la intención de diferenciar características distintivas de las empresas y la capacidad de ajuste organizacional ante la adopción de innovaciones, dado el estudio comparativo que aborda este trabajo al señalar que la capacidad de conocimiento en el aspecto organizacional es un elemento relevante para el éxito de la implementación de los sistemas de planificación empresariales.

a. Soporte de alta dirección.

El soporte de alta dirección es clave, tanto en la implementación del ERP, como en su asimilación (Kiriwandeniya et al., 2013). El soporte de alta dirección se puede denominar como la asistencia dinámica de los gerentes de nivel superior, hacia los de menor nivel, para proveer asesoramiento, esto con la finalidad de que los ejecutivos de menor nivel tengan la disposición para asignar los recursos adecuados para el buen funcionamiento y éxito del sistema ERP (Leyh, 2014), y por supuesto para que exista asimilación del ERP (Govindaraju, Hariadi y Sidiq, 2015). El soporte de alta dirección puede influir en las actitudes de los usuarios favoreciendo el uso del sistema ERP y estableciendo el alcance de la asimilación del ERP (Xu et al., 2017). Bajo estos argumentos se puede establecer la siguiente hipótesis:

$\mathrm{H}_{4}$ : el soporte de la alta dirección tiene un efecto positivo en la asimilación del ERP.

b. Ajuste organizacional.

Desde la etapa de la implementación, las organizaciones deben seleccionar un sistema ERP adecuado, es decir, que se ajuste a los procesos actuales en la empresa, como en los procesos comerciales llevados por el negocio (Law y Ngai, 2007), ya que las prácticas del sistema ERP pueden no ser compatibles con las existentes en la empresa (Morton y $\mathrm{Hu}, 2008$ ). En este sentido, las 
organizaciones que muestran un mayor ajuste organizacional, tienen más posibilidades de aumentar la asimilación del ERP (Xu et al., 2017). Con base en lo anterior, proponemos la siguiente hipótesis:

$\mathrm{H}_{5}$ : el ajuste organizacional tiene un efecto positivo en la asimilación del ERP.

c. Compromiso financiero.

De acuerdo a la literatura existente en sistemas de información (SI) y la implementación de tecnología, los recursos financieros son una pieza clave para las organizaciones (Zhu, Kraemer, Xu y Dedrick, 2004). Si la empresa cuenta con los conocimientos amplios para decidir cómo asignar de manera óptima los recursos, tanto para la capacitación de los usuarios, como para el mantenimiento y actualización del sistema ERP, incluyendo hardware y software, logrará fomentar la asimilación del sistema ERP (Xu et al., 2017) y poner de manifiesto el compromiso de la gerencia sobre la adopción del ERP (Zhu y Kraemer, 2005). Por lo tanto, habrá mayores posibilidades de aumentar la asimilación del ERP en aquellas organizaciones que poseen mayores recursos financieros comprometidos con el sistema ERP (Xu et al., 2017). Con base en lo anterior, se propone la siguiente hipótesis:

$\mathrm{H}_{6}$ : el compromiso financiero tiene un efecto positivo en la asimilación del ERP.

d. Transferencia de conocimiento.

La transferencia de conocimiento es un tema importante en la literatura de gestión (Lee y Lee, 2000) como un activo estratégico para las organizaciones (Vaghefi, Lapointe y Shahbaznezhad, 2018). Debido a que el conocimiento se encuentra en muchos niveles, su transferencia fluye del nivel individual a grupos y departamentos (Argote e Ingram, 2000), el conocimiento se transfiere cuando un cúmulo de conocimientos contenido en un individuo, en una tarea o técnica, se mueve de un grupo a otro, y el cúmulo de conocimientos del receptor se ve modificado (Reagans, Singh y Krishnan, 2015). En pocas palabras, la transferencia de conocimiento se refiere a la situación a través de la cual, una organización o individuo aprende de la experiencia de otro (Darr y Kurtzberg, 2000). En este sentido, para que haya transferencia de conocimiento, algunas organizaciones se apoyan en un grupo de usuarios con conocimiento y experiencia en el manejo de sistemas empresariales y luego éstos usuarios ayudan a otros a utilizar el nuevo sistema en sus operaciones (Volkoff, Elmes y Strong, 2004). Es importante resaltar que de acuerdo a investigaciones, se ha evidenciado que una organización puede mejorar su innovación haciendo uso de las habilidades de otros por medio de la transferencia de conocimiento (Pennings y Harianto, 1992). Además la comunicación efectiva entre los usuarios, permite que haya asimilación del sistema, al actualizar los conocimientos y mejorar la comprensión del sistema ERP (Kouki, Poulin y Pellerin, 2010). De lo expuesto anteriormente proponemos la siguiente hipótesis:

$\mathrm{H}_{7}$ : la transferencia de conocimiento tiene un efecto positivo en la asimilación del ERP. 


\subsubsection{Aspecto ambiental.}

Es preciso que los cambios ambientales se anticipen, monitoreen y evalúen, considerándolos en la toma de decisiones para prever ajustes en los requerimientos de los recursos ( Awa y Kalu, 2010), en el aspecto ambiental están comprendidos aquellos facilitadores e inhibidores operacionales, tales como la presión competitiva y el estímulo del gobierno (Al-Qirim, 2006). En este estudio se utiliza la presión competitiva como un factor relevante y una estrategia gerencial (Jeyaraj et al., 2006).

a. Presión competitiva.

La presión competitiva se define como la medida en que presionan a la empresa sus competidores (Xu et al., 2017). Estudios como el de Poston y Grabski (2001) y Vluggen (2005), han aceptado la presión competitiva como un factor clave y significativo que influye en el éxito y rendimiento del sistema ERP. Asimismo, investigaciones previas han señalado que la difusión de la innovación se ve aumentada por la presión competitiva del entorno (Zhu y Kraemer, 2005), puesto que, al sentir la presión de sus rivales, se inclinan con mayor facilidad a la adopción de las nuevas tecnologías (To y Ngai, 2006). Derivado de que los sistemas ERP se han convertido en sistemas estándar entre las organizaciones, la presión competitiva ejerce un fuerte estímulo a que las empresas asimilen el ERP más alto (Xu et al., 2017). De acuerdo con lo expuesto anteriormente se propone la siguiente hipótesis:

$\mathrm{H}_{8:}$ la presión competitiva tiene un efecto positivo en la asimilación del ERP.

Una vez descritas y desarrolladas las hipótesis consideradas en cada uno de los contextos: tecnológico, organizacional y ambiental, se desarrollan de igual forma las hipótesis referidas para evaluar el desempeño de la empresa, basadas en la teoría RBV, y resaltando que, únicamente cuando las empresas hagan uso del sistema ERP en la gestión operativa y comercial, incluyendo además aquellas actividades referidas a la cadena de valor, habrá un efecto de la asimilación del ERP en el desempeño de la empresa (Xu et al., 2017).

b. Desempeño financiero.

En la actualidad, las empresas esperan obtener beneficios tangibles al momento de implementar el sistema ERP, recuperando su inversión, reduciendo costos y maximizando ganancias (Abd et al., 2017). Existe un consenso de que el sistema ERP gestionado adecuadamente, provee beneficios tangibles trascendentes (Xu et al., 2017). Las organizaciones pueden tener un mayor desempeño financiero, a través de la asimilación del ERP, ya que al obtener una información precisa, ayuda a una mejor toma de decisiones, una gestión de efectivo óptima y alinear objetivos estratégicos (Sadrzadehrafiei et al., 2013), entre otros. Con base a lo anterior se propone la siguiente hipótesis:

$\mathrm{H}_{9}$ : el desempeño financiero tiene un efecto positivo en la asimilación del ERP. 
c. Desempeño no financiero.

Los beneficios intangibles de los sistemas de información gerencial, son difíciles de justificar en función de su rendimiento en términos económicos (Murphy y Simon, 2002). Es importante señalar que, el valor del ERP también se encuentra en características intangibles de la organización, como la satisfacción del cliente y el control de la gestión (Abd et al., 2017), es decir, el desempeño no financiero comprende los componentes del sistema empresarial: cliente, innovación y aprendizaje organizacional. En este sentido, por ejemplo, la asimilación del ERP puede mejorar la gestión del inventario y aumentar el ritmo de los negocios y aumentar la productividad de los empleados, que son parte del desempeño no financiero (Xu et al., 2017). Con base a lo anteriormente expuesto, se propone la siguiente hipótesis:

$\mathrm{H}_{10}$ : el desempeño no financiero tiene un efecto positivo en la asimilación del ERP.

\section{4. Metodología.}

\subsection{Diseño de investigación.}

La metodología empleada en este estudio es de carácter cuantitativo de tipo explicativo, no experimental y corte transversal. Esta investigación permitirá alcanzar el objetivo planteado, el cual es el de determinar el efecto de la asimilación de los sistemas de planificación de recursos empresariales y el desempeño en la industria restaurantera en el Noreste de México, distinguiendo las diferencias entre franquicias y empresas familiares, para examinar la causalidad de las variables del modelo de investigación vinculadas en el contexto TOE, ya que, en la industria restaurantera, ha sido poco abordado desde el enfoque de la asimilación del ERP.

\subsection{Muestreo y recolección de datos.}

La población objeto de estudio está compuesta por restaurantes que tengan hasta 250 empleados, y que en sus tareas rutinarias utilicen el sistema ERP. Como medida estratégica, se seleccionaron a través del Directorio Estadístico Nacional de Unidades Económicas (DENUE, 2019), posteriormente se realizó un muestreo por cuota de ocho municipios pertenecientes a Tamaulipas, resultando una muestra de 193 restaurantes, en los cuales se obtuvo una respuesta del $89.1 \%$, es decir se consideró una muestra de 172 de las cuales 70 son franquicias, representando un 40.7\% y 102 son empresas familiares, refiriendo un 59.3\%. Los datos se recopilaron mediante un cuestionario autoadministrado a gerentes o usuarios finales del sistema ERP y se recogieron respuestas entre noviembre 2019 y enero 2020. 


\subsection{Instrumento de medición.}

Para aumentar la validez y poner en práctica cada constructo, el modelo de investigación fue probado a través del método cuantitativo usando escalas previamente comprobadas, además los ítems se adaptaron de estudios empíricos, apoyados en la revisión de literatura, como se muestra en la tabla 1. Posteriormente se elaboró un primer borrador del cuestionario, como estudio piloto, donde se consideró la opinión de expertos y académicos y se probó previamente con 60 usuarios finales del ERP elegidos a conveniencia. La primera parte del cuestionario consideró una introducción con las instrucciones y el objetivo de este, y un conjunto de características generales de la empresa, tales como origen de la empresa (mexicana o extranjera), tipo de propiedad (franquicia o empresa familiar), entre otros. En la segunda parte, la construcción del modelo propuesto, el cual se midió utilizando una escala tipo Likert de 7 puntos, donde 1 corresponde a totalmente en desacuerdo y 7 a completamente de acuerdo.

Tabla 1

\section{Indicadores de las variables}

\begin{tabular}{lll}
\hline Variable/Código & Indicadores & Sustento Teórico \\
\hline Ventaja Relativa & & \\
\hline VR1 & El ERP aumenta la utilidad de la empresa & $\begin{array}{l}\text { Moore y Benbasat (1991); } \\
\text { Vluggen (2005) }\end{array}$ \\
VR2 & El ERP aumenta la eficiencia de la empresa & \\
VR3 & El ERP aumenta la competitividad de la empresa & \\
\hline Compatibilidad & & Moore y Benbasat (1991); \\
\hline CTB1 & El ERP complementa el sistema de la empresa & Vluggen (2005) \\
CTB2 & El ERP se adapta a las necesidades de la empresa & \\
CTB3 & El ERP es compatible con el funcionamiento general de & \\
\hline Complejidad & la empresa & \\
\hline CJ1 & Aprender a usar el ERP ha sido fácil para los empleados & $\begin{array}{l}\text { Moore y Benbasat (1991); } \\
\text { Vluggen (2005) }\end{array}$ \\
CJ2 & El mantenimiento del ERP es sencillo & \\
CJ3 & El ERP es fácil de operar &
\end{tabular}

Soporte de Alta Dirección

SOP1 La implementación del ERP recibió apoyo de los Liang et al. (2007); Zhu et directivos al. (2010)

SOP2

Los Directivos participaron en la implementación del ERP 
Los gerentes establecieron metas y estándares para monitorear el proyecto ERP

\begin{tabular}{|c|c|c|}
\hline \multicolumn{3}{|c|}{ Ajuste Organizacional } \\
\hline $\mathrm{AO} 1$ & $\begin{array}{l}\text { Se realizó una reingeniería apropiada del proceso de } \\
\text { negocios antes de la implementación }\end{array}$ & $\begin{array}{l}\text { Zhu et al. (2010); Hong y } \\
\text { Kim (2002) }\end{array}$ \\
\hline $\mathrm{AO} 2$ & $\begin{array}{l}\text { Los usuarios finales recibieron capacitación y educación } \\
\text { sobre ERP y sus aplicaciones }\end{array}$ & \\
\hline $\mathrm{AO} 3$ & $\begin{array}{l}\text { La funcionalidad integrada en ERP satisface las } \\
\text { necesidades requeridas por nuestra empresa }\end{array}$ & \\
\hline $\mathrm{AO} 4$ & $\begin{array}{l}\text { El flujo de procesos elaborado en el sistema ERP } \\
\text { corresponde al flujo de procesos de nuestra empresa }\end{array}$ & \\
\hline AO5 & $\begin{array}{l}\text { La interfaz del usuario está diseñada para la toma de } \\
\text { decisiones de nuestra empresa }\end{array}$ & \\
\hline
\end{tabular}

Compromiso Financiero

\begin{tabular}{lll}
\hline CF1 & $\begin{array}{l}\text { Los ingresos de la empresa permiten implementar un } \\
\text { sistema ERP }\end{array}$ & Zhu y Kraemer (2005) \\
CF2 & $\begin{array}{l}\text { El gasto de la implementación del ERP es menor a los } \\
\text { ingresos de la empresa }\end{array}$ & \\
\hline
\end{tabular}

\section{Transferencia de Conocimiento}

\section{TC1 mentor para apoyarlo en su desarrollo dentro de la empresa}

TC2

TC3

TC4

TC5

TC6

\section{Presión Competitiva}

Gran parte del conocimiento se distribuye de manera informal

Se organizan reuniones periódicas en las que se discuten asuntos profesionales

Los empleados comparten con sus compañeros sobre las experiencias positivas y proyectos exitosos

Se tiene un formulario de revisión interna, en el cual los miembros discuten sus métodos de trabajo

Los miembros cambian de trabajo regularmente, distribuyendo así su know-how
Filius, de Jong y Roelofs

(2000)

$\begin{array}{ll}\text { PC1 } & \begin{array}{l}\text { La empresa enfrenta un alto nivel de rivalidad entre las } \\ \text { empresas del mismo giro }\end{array} \\ \text { PC2 } & \begin{array}{l}\text { La empresa enfrenta un alto nivel de presión por parte de } \\ \text { la industria sobre el uso del ERP como un estándar } \\ \text { industrial }\end{array}\end{array}$

Vluggen (2005); Zhu y

Kraemer (2005);

Gangwar, Date y

Ramaswamy (2015)

\footnotetext{
industrial
} 


$\begin{array}{ll}\text { PC3 } & \begin{array}{l}\text { Somos conscientes de la implementación del sistema } \\ \text { ERP en las empresas competidoras }\end{array} \\ \text { PC4 } & \begin{array}{l}\text { Entendemos la ventaja competitiva que ofrecen los } \\ \text { sistemas ERP }\end{array} \\ \text { PC5 } & \begin{array}{l}\text { El uso del ERP en los competidores de la empresa afecta } \\ \text { el alcance de mercado }\end{array}\end{array}$

Asimilación del ERP

\begin{tabular}{lll} 
AS1 & $\begin{array}{l}\text { El sistema ERP controla la gestión financiera de la } \\
\text { empresa }\end{array}$ & $\begin{array}{l}\text { Karimi et al. (2007); } \\
\text { Liang et al. (2007); Lin y } \\
\text { Lin (2008) }\end{array}$ \\
AS2 & $\begin{array}{l}\text { El sistema ERP controla la gestión logística de la } \\
\text { empresa }\end{array}$ & \\
AS3 & $\begin{array}{l}\text { El sistema ERP controla la gestión de recursos humanos } \\
\text { de la empresa }\end{array}$ \\
\hline
\end{tabular}

Desempeño Financiero

\begin{tabular}{|c|c|c|}
\hline DF1 & $\begin{array}{l}\text { La asimilación del ERP ha aumentado la tasa de rotación } \\
\text { de inventario }\end{array}$ & $\begin{array}{l}\text { Hitt et al. (2002); Hunton } \\
\text { et al. (2003); Nicolau } \\
\text { (2004) }\end{array}$ \\
\hline DF2 & $\begin{array}{l}\text { La asimilación del ERP ha aumentado la tasa de rotación } \\
\text { de cuentas por cobrar }\end{array}$ & \\
\hline DF3 & $\begin{array}{l}\text { La asimilación del ERP ha reducido la tasa de costo de } \\
\text { ventas }\end{array}$ & \\
\hline DF4 & $\begin{array}{l}\text { La asimilación del ERP ha aumentado el rendimiento de } \\
\text { los activos }\end{array}$ & \\
\hline DF5 & $\begin{array}{l}\text { La asimilación del ERP ha aumentado la tasa de } \\
\text { ganancia de ventas }\end{array}$ & \\
\hline DF5 & $\begin{array}{l}\text { La asimilación del ERP ha aumentado el retorno de la } \\
\text { inversión }\end{array}$ & \\
\hline \multicolumn{3}{|c|}{ Desempeño no Financiero } \\
\hline DNF1 & $\begin{array}{l}\text { La asimilación del ERP ha reducido las quejas de los } \\
\text { clientes }\end{array}$ & $\begin{array}{l}\text { Grover y Davenport } \\
(2001)\end{array}$ \\
\hline DNF2 & $\begin{array}{l}\text { La asimilación del ERP ha aumentado la satisfacción de } \\
\text { los clientes }\end{array}$ & \\
\hline DNF3 & $\begin{array}{l}\text { La asimilación del ERP ha aumentado la calidad del } \\
\text { proceso interno }\end{array}$ & \\
\hline DNF4 & $\begin{array}{l}\text { La asimilación del ERP ha reducido el tiempo de ciclo } \\
\text { del proceso }\end{array}$ & \\
\hline DNF5 & La asimilación del ERP ha reducido el costo del proceso & \\
\hline DNF6 & $\begin{array}{l}\text { La asimilación del ERP ha aumentado el intercambio de } \\
\text { conocimientos de los trabajadores }\end{array}$ & \\
\hline DNF7 & $\begin{array}{l}\text { La asimilación del ERP ha aumentado la productividad } \\
\text { de los empleados }\end{array}$ & \\
\hline
\end{tabular}

Fuente: elaboración propia basada en Xu, Ou y Fan (2017) y Filius, de Jong y Roelofs (2000). 


\section{$\rightarrow$ 5. Resultados.}

Para el análisis de los datos se utilizó el software Smart PLS versión 3.2.8 (Ringle, Wende y Becker, 2015), ya que este software posibilita evaluar el modelo de investigación, tanto por grupos, como de manera completa (Hair, Hult, Ringle y Sarstedt, 2017; Henseler, Ringle, y Sarstedt, 2016). Dentro de los datos descriptivos importantes en nuestra investigación, podemos mencionar que, de la muestra de 172 restaurantes, el $40.7 \%$ está conformada por franquicias y el 59.3\% por empresas familiares. Cabe mencionar que, se realizó un método de sesgo común utilizando la prueba de un factor de Harman, para verificar que ningún factor explique individualmente la mayoría de la varianza; el resultado que se obtuvo fue la presencia de un solo factor que explica un $27.98 \%$ de la varianza y, como este valor es menor al 50\% indica que no hay sesgo (Podsakoff, Mackenzie, Lee y Podsakoff, 2003). Además, se consideró el tamaño de la muestra lo suficientemente grande y adecuada para realizar pruebas estadísticas (Cohen, 1992).

\subsection{Valoración del Modelo de Medida.}

Se utilizó el método de modelado de ecuaciones estructurales (SEM) con mínimos cuadrados parciales (PLS) (Hair, Ringle y Sarstedt, 2011) para analizar la relación y los efectos causales del modelo propuesto (Figura 1), considerándolo adecuado, ya que es el más utilizado cuando se trata de analizar las relaciones causa-efecto entre constructos latentes (Hair et al., 2011). PLS requiere muestras más pequeñas y reduce las variaciones residuales de las construcciones endógenas, siendo común para estimar modelos teóricos y contextos de datos empíricos (Hair, Ringle y Sarstedt, 2013; Henseler y Chin, 2010).

La fiabilidad individual del ítem, se realizó a través del análisis de las cargas $(\lambda)$, las cuales varían entre 0 y 1 , y en el caso particular de los modelos reflectivos, como es el caso de nuestro modelo propuesto, las cargas deben ser mayores a 0.707 , describiendo de esta manera, el $50 \%$ de la varianza del indicador explicada por su factor (Hair, Hult, Ringle y Sarstedt, 2017). Cabe aclarar que algunos de los valores que no cumplieron con la condición anterior, fueron eliminados reportando solo los valores de carga aceptable (tabla 2).

Con respecto a la fiabilidad del constructo, esta se refiere a que tan estricta es la medición de las variables manifiestas con la misma variable latente, considerándose valores permisibles de Alfa de Cronbach $(\alpha)$ y fiabilidad compuesta $(\rho c)$ mayores a 0.7 ( Hair, Anderson, Tatham y Black, 2007; Segars, 1997). Los valores resultantes se muestran en la tabla 2, cumpliendo con esta condición, por lo que se consideran aceptables. En cuanto a la validez convergente, se evaluó mediante la varianza extraída media (AVE), este valor debe ser mayor a 0.50 (Chin, 1998) y se refiere al hecho de que un 
conjunto de indicadores represente un valor único del constructo subyacente (Henseler, 2017). De acuerdo con los resultados presentados (tabla 2), se puede deducir que todos los valores cumplen con la validez convergente. Por último, la validez discriminante se evaluó con la ratio HeterotraitMonotrait (HTMT) donde todos los valores deben ser inferiores a 0.90 (Henseler, Hubona y Ray, 2016), en el análisis, todos los constructos cumplen con la condición de ser menores a 0.90 (tabla 3).

Tabla 2

Fiabilidad del ítem y validez convergente

\begin{tabular}{l} 
Variables/Ítems \\
\hline Ventaja Competitiva (VR) \\
El ERP aumenta la utilidad de la empresa \\
El ERP aumenta la eficiencia de la empresa \\
El ERP aumenta la competitividad de la \\
empresa
\end{tabular}

Compatibilidad (CTB)

El ERP complementa el sistema de la empresa

El ERP se adapta a las necesidades de la empresa

El ERP es compatible con el funcionamiento general de la empresa

\section{Complejidad (CJ)}

Aprender a usar el ERP ha sido fácil para los empleados

El mantenimiento del sistema ERP es sencillo

El ERP es fácil de operar

\section{Soporte de Alta Dirección (AD)}

La implementación de ERP recibió apoyo de los directivos

Los directivos participaron en la implementación de ERP

Los gerentes establecieron metas y estándares para monitorear el proyecto ERP

\section{Ajuste de la Organización (AO)}

Los usuarios finales recibieron capacitación y educación sobre ERP y sus aplicaciones

La funcionalidad integrada en ERP satisface las necesidades requeridas por nuestra empresa
$\lambda$
0.791
0.84
0.888

0.808

$\begin{array}{cccc}\text { Rho_A } & \text { pc } & \text { AVE } & \text { R }^{2} \\ 0.927 & 0.878 & 0.707 & \text { NA }\end{array}$

$\begin{array}{lllll}0.779 & 0.782 & 0.872 & 0.694 & \text { NA }\end{array}$

0.799

0.874

0.825

$\begin{array}{lllll}0.887 & 0.914 & 0.929 & 0.814 & \text { NA }\end{array}$

0.928

0.878

0.899

$\begin{array}{lllll}0.805 & 0.863 & 0.883 & 0.716 & \text { NA }\end{array}$

0.815

0.912

0.808
0.82
0.829
0.881
0.649
NA

0.814

0.771 
El flujo de procesos elaborado en el sistema ERP corresponde al flujo de procesos de nuestra empresa

La interfaz del usuario de ERP está diseñada para la toma de decisiones de nuestra empresa

\section{Compromiso Financiero (CF)}

Los ingresos de la empresa permiten implementar un sistema ERP

El gasto de la implementación de ERP es menor a los ingresos de la empresa

\section{Transferencia de Conocimiento (TC)}

A un nuevo miembro de la empresa se le asigna un mentor para apoyarlo en su desarrollo dentro de la empresa

Se organizan reuniones periódicas en las que se discuten asuntos profesionales

Los empleados comparten con sus compañeros sobre las experiencias positivas y proyectos exitosos

Se tiene un formulario de revisión interna, en el cual los miembros discuten sus métodos de trabajo

\section{Presión Competitiva (PC)}

La empresa enfrenta un alto nivel de rivalidad entre las empresas del mismo giro o industria

Somos conscientes de la implementación del sistema ERP en las empresas competidoras

Entendemos la ventaja competitiva que ofrecen los sistemas ERP

\section{Asimilación de ERP (AS)}

El sistema ERP controla la gestión financiera de la empresa

El sistema ERP controla la gestión logística de la empresa

El sistema ERP controla la gestión de recursos humanos de la empresa

\section{Desempeño Financiero (DF)}

La asimilación del ERP ha aumentado la tasa de rotación de inventario

La asimilación del ERP ha reducido la tasa de costo de ventas

La asimilación del ERP ha aumentado el rendimiento de los activos

La asimilación del ERP ha aumentado la tasa de ganancia de ventas
0.763
0.733
0.792
0.879
0.785
NA

0.845

0.925

$\begin{array}{lllll}0.887 & 0.902 & 0.921 & 0.745 & \text { NA }\end{array}$

0.834

0.86
0.717
0.736
0.839
0.636
NA

0.783

0.861

0.743
0.856
0.86
$0.913 \quad 0.777$
0.329

0.906

0.901

0.836

$\begin{array}{lllll}0.829 & 0.833 & 0.886 & 0.662 & 0.206\end{array}$


Desempeño no Financiero (DNF)

La asimilación dle ERP ha aumentado la calidad del proceso interno

La asimilación del ERP ha reducido el tiempo

del ciclo del proceso

La asimilación del ERP ha reducido el costo del

proceso

La asimilación del ERP ha aumentado el

intercambio de conocimientos de los

trabajadores

La asimilación del ERP ha aumentado la productividad de los empleados

$\begin{array}{lllll}0.838 & 0.863 & 0.881 & 0.597 & 0.184\end{array}$

0.722

0.743

0.78

0.785

0.829

Fuente: elaboración propia con base en las salidas del software Smart PLS 3.2.8.

Habiendo verificado que las medidas de los constructos son fiables y válidas, se procedió a la valoración de los resultados del modelo estructural, donde se examina el poder de predicción del modelo y las relaciones entre los constructos (Hair et al., 2019).

Tabla 3

Validez Discriminante HTMT

\begin{tabular}{lccccccccccc}
\hline Variables & AO & AS & CF & CTB & CJ & DF & DNF & PC & SOP & TC & VR \\
\hline Ajuste Organizacional (AO) & & & & & & & & & & & \\
Asimilación del ERP (AS) & 0.388 & & & & & & & & & \\
Compromiso Financiero (CF) & 0.614 & 0.476 & & & & & & & & \\
Compatibilidad (CTB) & 0.679 & 0.271 & 0.389 & & & & & & & \\
Complejidad (CJ) & 0.491 & 0.523 & 0.595 & 0.438 & & & & & & \\
Desempeño Financiero (DF) & 0.536 & 0.539 & 0.436 & 0.308 & 0.293 & & & & & \\
Desempeño no Financiero & & & & & & & & & & \\
(DNF) & 0.684 & 0.485 & 0.702 & 0.465 & 0.58 & 0.689 & & & & \\
Presión Competitiva (PC) & 0.536 & 0.455 & 0.483 & 0.536 & 0.318 & 0.533 & 0.484 & & & \\
Soporte Alta Dirección (SOP) & 0.634 & 0.326 & 0.465 & 0.661 & 0.415 & 0.266 & 0.565 & 0.485 & & & \\
Transferencia de & & & & & & & & & & \\
Conocimiento (TC) & 0.484 & 0.526 & 0.586 & 0.45 & 0.458 & 0.312 & 0.617 & 0.402 & 0.617 & \\
Ventaja Relativa (VR) & 0.519 & 0.243 & 0.471 & 0.78 & 0.458 & 0.35 & 0.502 & 0.506 & 0.69 & 0.403 \\
\hline
\end{tabular}

Fuente: elaboración propia con base en las salidas del software Smart PLS 3.2.8.

\subsection{Valoración del Modelo Estructural.}

El modelo de investigación se analizó examinando el signo, la magnitud y la varianza explicada $\left(\mathrm{R}^{2}\right)$ de las variables dependientes, los valores de $\mathrm{R}^{2}$ de $0.19,0.35$ y 0.67 , son considerados como débil, medio y sustancial (Henseler, Ringle y Sinkovics, 2009), con base en este criterio, se 
puede deducir que los constructos dependientes tienen un valor predictivo adecuado (tabla 2) y se puede constatar por medio de la varianza explicada $\left(\mathrm{R}^{2}\right)$. Además de evaluar $\mathrm{R}^{2}$, también se examinó el valor del indicador Stone-Geiser $\left(\mathrm{Q}^{2}\right)$, el cual muestra el poder predictivo fuera de la muestra (Henseler et al., 2009), donde los valores propuestos deben ser mayores que cero, 0.25 y 0.50 respectivamente, condiciones de pequeña, media y gran relevancia predictiva del modelo (Hair, Risher, Sarstedt y Ringle, 2019). Los valores obtenidos del análisis cumplen con los requerimientos, demostrando con ello la relevancia predictiva del nomograma (tabla 4).

Tabla 4

\section{Relevancia Predictiva}

\begin{tabular}{lccc}
\hline Variable & SSO & SSE & Q $^{\mathbf{2}}$ (=1-SSE/SSO) \\
\hline Ventaja Relativa & 516 & 516 & \\
Compatibilidad & 516 & 516 & \\
Complejidad & 516 & 516 & \\
Soporte Alta Dirección & 516 & 516 & \\
Ajuste Organizacional & 688 & 688 & \\
Compromiso Financiero & 344 & 344 & \\
Transferencia de Conocimiento & 688 & 688 & \\
Presión Competitiva & 516 & 516 & \\
Asimilación del ERP & 516 & 397.457 & 0.230 \\
Desempeño Financiero & 688 & 603.676 & 0.123 \\
Desempeño no Financiero & 860 & 776.349 & 0.097 \\
\hline
\end{tabular}

Fuente: elaboración propia con base en las salidas del software Smart PLS 3.2.8.

Posteriormente se realizó un bootstraping no paramétrico, con 172 casos y 5000 submuestras. La importancia del bootstraping reside en analizar los valores de las rutas estructurales y, por ende, la aceptación o no de la hipótesis, a través de los resultados del error estándar y los valores t de Student de los parámetros del modelo (Henseler, Hubona y Ray, 2016). En la tabla 5 se presentan los resultados obtenidos, para evaluar la precisión y la estabilidad de las estimaciones; cabe resaltar que la variable transferencia de conocimiento es la que más aporta al modelo, mientras que la variable presión competitiva es la que aporta menos. Cabe mencionar que la complejidad, ha sido rechazada, por el signo contrario al considerado a la hipótesis formulada. 
Tabla 5

\section{Resultados del Modelo Estructural}

\begin{tabular}{lccc}
\hline Hipótesis & $\boldsymbol{\beta}$ & Valores t & Observaciones \\
\hline H1: Ventaja Relativa $\rightarrow$ Asimilación del ERP & -0.042 & 0.294 & Rechazada \\
H2: Compatibilidad $\rightarrow$ Asimilación del ERP & -0.076 & 0.767 & Rechazada \\
H3: Complejidad $\rightarrow$ Asimilación del ERP & 0.300 & $3.028^{* *}$ & Aceptada \\
H4: Soporte Alta Dirección $\rightarrow$ Asimilación del ERP & -0.038 & 0.321 & Rechazada \\
H5: Ajuste Organizacional $\rightarrow$ Asimilación del ERP & 0.048 & 0.483 & Rechazada \\
H6: Compromiso Financiero $\rightarrow$ Asimilación del ERP & 0.055 & 0.499 & Rechazada \\
H7: Transferencia de conocimiento $\rightarrow$ Asimilación del ERP & 0.295 & $2.703^{* *}$ & Aceptada \\
H8: Presión Competitiva $\rightarrow$ Asimilación del ERP & 0.220 & $1.707^{*}$ & Aceptada \\
H9: Desempeño Financiero $\rightarrow$ Asimilación del ERP & 0.459 & $5.918^{* * *}$ & Aceptada \\
H10: Desempeño No Financiero $\rightarrow$ Asimilación del ERP & 0.434 & $5.131^{* * *}$ & Aceptada \\
\hline
\end{tabular}

Fuente: elaboración propia con base salida software Smart PLS 3.2.8.

\subsection{Tipo de empresa como variable moderadora.}

En este apartado se incluye el tipo de empresa en el modelo final obtenido, para examinar si el tipo de empresa afecta el desempeño en los dos grupos. Para tal objetivo se ha vuelto a ejecutar el algoritmo para cada uno de los dos tipos de empresas: empresas familiares y franquicias. Es importante destacar que el modelo propuesto completo (figura 2), explica el 32.9\% de la varianza, mientras que el modelo de las empresas familiares explica el $35.6 \%$ y por su parte el modelo de las franquicias, con la intervención de la variable Asimilación del ERP, con un valor ligeramente menor explica un $29.5 \%$ de la varianza. Otro dato notable es la importancia dada a cada una de las variables en cada grupo, por ejemplo, para las empresas familiares, la transferencia de conocimiento y la presión competitiva tienen mayor peso, mientras que para las franquicias son el soporte de la alta dirección y el compromiso financiero. Además, la asimilación del ERP en el modelo completo explica un $20.6 \%$ del desempeño financiero y un $18.4 \%$ del desempeño no financiero, y en el caso de las empresas familiares la asimilación del ERP explica un 29.8\% del rendimiento financiero y un $24 \%$ del no financiero, mientras que, para el modelo de las franquicias, la asimilación del ERP solo explica un $8.4 \%$ del desempeño financiero y un $11.1 \%$ del desempeño no financiero.

Finalmente, se llevó a cabo un análisis multigrupo para comparar los resultados de acuerdo con el tipo de empresa (empresa familiar y franquicia) con la finalidad de comprobar que las diferencias entre los distintos tipos de empresas no sean debidas al contenido del modelo de medida o al modelo estructural, para esto primero hay que cumplir con ciertas consideraciones, que se detallan a continuación. 
Tipo de empresa en el modelo de trayectoria PLS-SEM propuesto
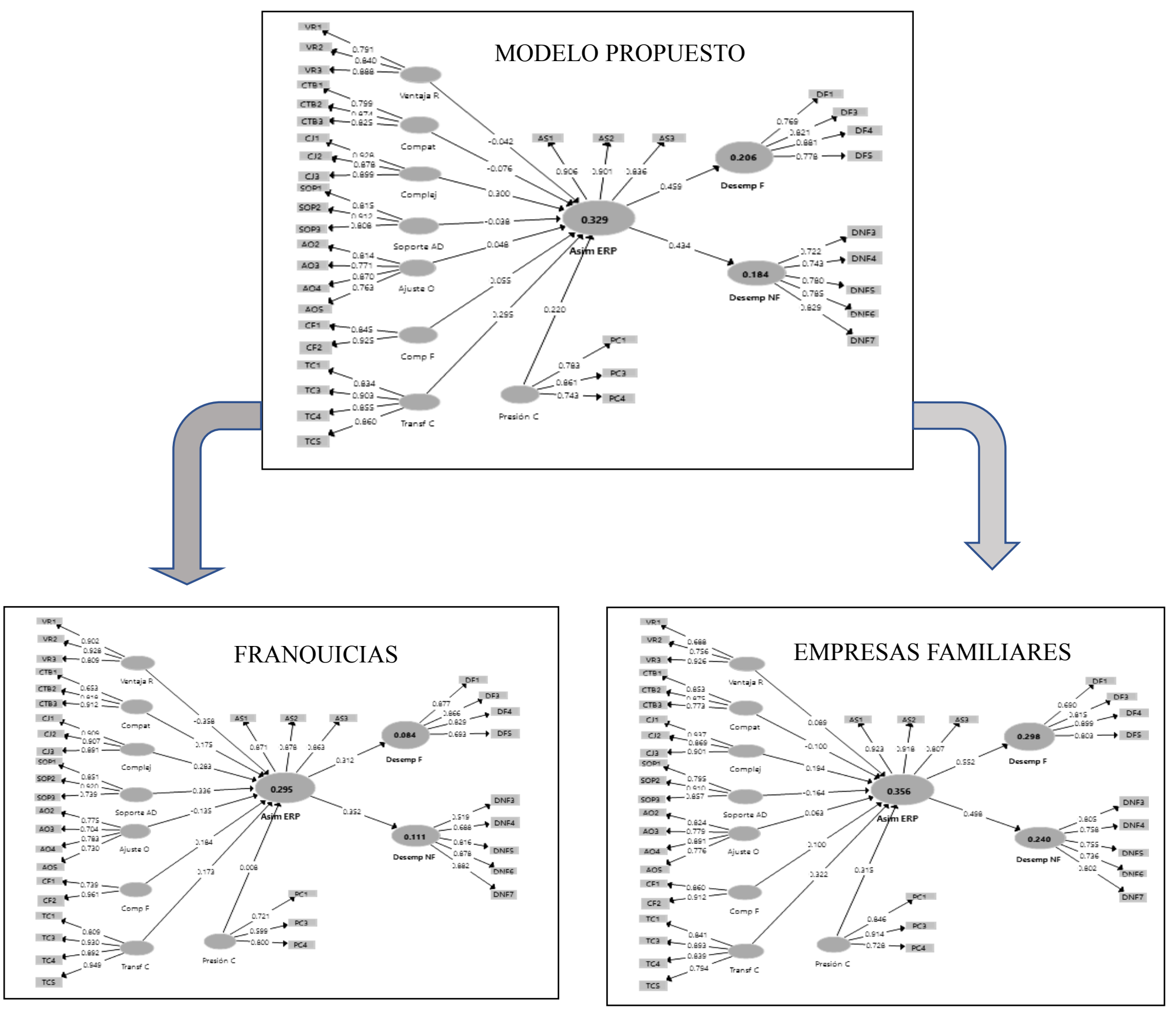


\subsection{Evaluación de la invarianza de la medición.}

Se llevó a cabo el procedimiento MICOM (Invarianza de Medición de Modelos Compuestos) para analizar que las diferencias entre ambos grupos no sean derivadas del contenido, ya sea del modelo de medición o el modelo estructural (Henseler et al., 2016). Siguiendo a Hair et al. (2019), el procedimiento MICOM consta de tres pasos: 1) invarianza de configuración, 2) invarianza de compuesto y 3 ) igualdad de medias y varianzas de los compuestos. En el paso 1, en relación con la invarianza de configuración, ambos modelos (franquicias y empresa familiar) tienen los mismos indicadores, siguen el mismo tratamiento e igual algoritmo, por lo que se cumple con la invarianza de configuración. En el paso 2, se establece que existe una invarianza de compuesto, ya que los valores de correlación están por arriba del percentil 5\% y, además, los valores de $\mathrm{p}$ son mayores a 0.05. En el paso 3, deben evaluarse tanto la igualdad de las medias de los compuestos, como la igualdad de varianzas. En la tabla 6, se presentan los resultados observando que no se cumple con ambas igualdades, sin embargo, se confirma una invarianza de medida parcial, la cual permite realizar las comparaciones de los valores de coeficientes estandarizados del modelo estructural entre los compuestos de los dos grupos (franquicias y empresas familiares).

Tabla 6

\section{Invarianza de la medición}

\begin{tabular}{lccccccccccccc}
\hline & Paso 1 & \multicolumn{3}{c}{ Paso } & \multicolumn{1}{c}{ P } & \multicolumn{1}{c}{ Paso 3 } \\
\hline Variables & ¿IC? & Co & $\mathbf{0 . 0 5}$ & ;CIE? & DMV & $\mathbf{0 . 0 5}$ & $\mathbf{0 . 9 5}$ & ¿Cumple? & VC & $\mathbf{0 . 0 5}$ & $\mathbf{0 . 9 5}$ & ¿Cumple? \\
\hline VR & Sí & 0.919 & 0.768 & Sí & 0.22 & -0.25 & 0.266 & Sí & -0.063 & -0.479 & 0.467 & Sí \\
CTB & Sí & 0.931 & 0.877 & Sí & 0.054 & -0.25 & 0.258 & Sí & -0.261 & -0.593 & 0.533 & Sí \\
CJ & Sí & 0.998 & 0.996 & Sí & 0.355 & -0.25 & 0.258 & No & -0.612 & -0.429 & 0.37 & No \\
SOP & Sí & 0.995 & 0.954 & Sí & -0.04 & -0.27 & 0.262 & Sí & 0.222 & -0.458 & 0.476 & Sí \\
AO & Sí & 0.993 & 0.959 & Sí & 0.347 & -0.26 & 0.252 & No & -0.72 & -0.439 & 0.423 & No \\
CF & Sí & 0.988 & 0.98 & Sí & 0.489 & -0.26 & 0.267 & No & -0.631 & -0.342 & 0.286 & No \\
TC & Sí & 0.998 & 0.985 & Sí & 0.262 & -0.27 & 0.275 & Sí & -0.003 & -0.444 & 0.39 & Sí \\
PC & Sí & 0.974 & 0.904 & Sí & 0.259 & -0.27 & 0.262 & Sí & -0.571 & -0.544 & 0.508 & No \\
AS & Sí & 0.999 & 0.991 & Sí & 0.345 & -0.27 & 0.258 & No & -0.558 & -0.678 & 0.612 & Sí \\
DF & Sí & 0.99 & 0.95 & Sí & 0.232 & -0.25 & 0.246 & Sí & 0.18 & -0.566 & 0.497 & Sí \\
DNF & Sí & 0.993 & 0.935 & Sí & 0.153 & -0.27 & 0.249 & Sí & 0.102 & -0.461 & 0.416 & Sí \\
\hline
\end{tabular}

Nota: IC: Invarianza Configural; Co: Invarianza correlacional; CIE: Invarianza de Compuesto Comprobada establecida; DMV: Diferencia valor medio; VC: Igualdad de Varianzas Comprobada.

Fuente: elaboración propia con base en las salidas del software Smart PLS 3.2.8. 
Una vez efectuado el análisis MICOM, se procede a realizar el análisis multigrupo, para este objetivo se aplica un análisis basado en permutaciones, el análisis MGA (Sarstedt, Henseler, y Ringle, 2011), así como la prueba paramétrica. Los resultados muestran diferencias significativas en la asimilación del ERP, entre los grupos, de forma concreta, en la relación existente entre el soporte de alta dirección y la asimilación del ERP, así como también en la ventaja relativa y la asimilación del ERP (tabla 7).

Tabla 7

Resultados de las Pruebas Multigrupos

\begin{tabular}{lccccc}
\hline \multirow{2}{*}{ Relaciones } & \multicolumn{2}{c}{ Prueba de Permutación } & MGA & $\begin{array}{c}\text { Prueba } \\
\text { Paramétrica }\end{array}$ \\
\cline { 2 - 6 } & Franquicias & Emp Fam & Valor $\mathrm{p}$ & Valor $\mathrm{p}$ & Valor $\mathrm{p}$ \\
\hline Ventaja $\mathrm{R} \rightarrow$ Asim ERP & -0.381 & 0.091 & 0.033 & 0.959 & 0.045 \\
Compat $\rightarrow$ Asim ERP & 0.18 & -0.096 & 0.085 & 0.155 & 0.132 \\
Complej $\rightarrow$ Asim ERP & 0.264 & 0.192 & 0.364 & 0.368 & 0.374 \\
Soporte AD $\rightarrow$ Asim & 0.356 & -0.17 & 0.025 & 0.01 & 0.007 \\
ERP & & & & & \\
Ajuste Org $\rightarrow$ & -0.141 & 0.061 & 0.182 & 0.823 & 0.176 \\
Asim ERP & 0.176 & 0.101 & 0.432 & 0.353 & 0.365 \\
Comp F $\rightarrow$ Asim ERP & 0.175 & 0.322 & 0.315 & 0.779 & 0.226 \\
Transf C $\rightarrow$ Asim ERP & 0.023 & 0.316 & 0.154 & 0.911 & 0.096 \\
Presión C $\rightarrow$ Asim ERP & 0.33 & 0.555 & 0.068 & 0.93 & $\mathbf{0 . 0 5 7}$ \\
Asim ERP $\rightarrow$ DF & 0.365 & 0.5 & 0.215 & 0.786 & 0.204 \\
Asim ERP $\rightarrow$ DNF & & & & & \\
\hline
\end{tabular}

Fuente: elaboración propia con base en las salidas del software Smart PLS 3.2.8

\subsection{Discusión de los resultados.}

Esta investigación utilizó el marco TOE (tecnología-organización-medio ambiente) para analizar el efecto de los factores que influyen en la asimilación del ERP, utilizando un conjunto de datos del sector restaurantero, probando los efectos de ocho factores. Los resultados empíricos se discuten a continuación.

\subsubsection{Contexto Tecnológico.}

La variable ventaja relativa (H1) no afecta significativamente a la asimilación del ERP, un resultado opuesto a la hipótesis formulada, sin embargo, esto va en línea con Wang et al. ( 2016) y AL-Shboul (2018), pudiera deberse a que el dominio de la aplicación del sistema de gestión, no se ha alcanzado sino hasta que los usuarios hayan logrado la habilidad en el manejo de la aplicación. Sin 
embargo contradice los resultados de otros investigadores ( $\mathrm{Xu}$ et al., 2017). En cuanto a la compatibilidad (H2), los datos indicaron que la compatibilidad no presenta significación en la asimilación del sistema ERP, un resultado opuesto a la hipótesis formulada, pero estos resultados son consistentes con Albar y Hoque (2017) e Ifinedo (2011), y contradictorios a AL-Shboul (2018) y Ruivo et al. (2013).Tal vez porque las empresas medianas o pequeñas, no perciben que el ERP sea consistente con sus prácticas y especulan que tales tecnologías no cubren con las necesidades propias de la empresa. Por último, en el contexto tecnológico tenemos a la variable complejidad (H3), la cual se esperaba un efecto negativo en la asimilación del ERP, sin embargo, en el análisis resulta significativa, pero con signo positivo, por lo que dicha hipótesis es rechazada, en línea con Ahani, Rahim y Nilashi (2017), esto podría deberse a que como hay muchas empresas que no están familiarizadas con el sistema ERP (Chang, Hung y Yen, 2010), las hace depender de los proveedores y consultores, es decir de apoyo externo para resolver dudas de operación o mantenimiento, a cambio del precio alto del sistema de gestión, percibiendo dicho sistema como una herramienta útil en la asimilación, esto es, mientras más complejo lo perciban, mayores beneficios consideran recibir, por la cantidad de áreas que integra.

\subsubsection{Contexto Organizacional.}

En el contexto organizacional el soporte de alta dirección (H4), los resultados de los análisis mostraron que no hay relación significativa con la asimilación del ERP, esto puede deberse a la falta de apoyo de la misma, ya sea por desconocimiento de los beneficios y del funcionamiento del sistema ERP, los resultados son consistentes con algunos estudios anteriores (Bradford y Florin, 2003; Mangula et al., 2014). Otra variable de este contexto es el ajuste organizacional (H5), que de acuerdo a los resultados, fue no significativa en la asimilación del ERP, una de las razones principales es que, según evidencias de Pudjianto et al. (2011), en los países en desarrollo, el ajuste organizacional no es significativo en la asimilación de TI. Los resultados fueron coherentes con diversos autores (Mangula et al., 2014; Pudjianto et al., 2011) asumiendo un enfoque distinto al de este estudio, que se esperaba distinguiera la investigación prevaleciendo el enfoque orientado al desempeño. También tenemos el compromiso financiero (H6), que de acuerdo a los resultados resultó ser no significativa, esperando un efecto contrario en la formulación inicial, similar al resultado de Ifinedo (2011). Esto podría deberse esencialmente a que como las Pymes no cuentan con un presupuesto financiero destinado para invertir en un sistema ERP o cualquier otra innovación, sino están más preocupados por recuperar la inversión inicial, por ende, no es significativo. Por último, tenemos a la variable transferencia de conocimiento (H7), que es un hallazgo importante, ya que fue la única significativa en este contexto y es la variable que se incorporó al modelo. La transferencia de conocimiento inicia desde la implementación del sistema ERP, ya que la mayoría de los sistemas incluyen modelos de 
referencia de procesos comerciales, los cuales al asignarse al negocio de una empresa, el conocimiento del proceso es transferido (Lee y Lee, 2000), siendo congruente con investigaciones anteriores (Hung et al., 2012; Low y Robins, 2014). Es importante mencionar que de acuerdo a estudios previos, la transferencia es más efectiva cuando se tiene una relación de interdependencia y estandarización como en la franquicia (Darr, Argote y Epple, 1995). Además en un inicio el conocimiento especializado puede provenir de un consultor externo, sin embargo, es trascendental que haya transferencia de conocimiento desde el equipo interno de la organización (Goyette, Cassivi, Courchesne y Elia, 2015), para que en la etapa de asimilación ésta sea efectiva.

\subsubsection{Contexto Ambiental.}

En este contexto solo tenemos una variable, que es la presión competitiva (H8), y de acuerdo con los resultados, es significativa en la asimilación del ERP, pudiera deberse a que, a medida que el sistema ERP adquiere familiaridad y popularidad, las Pymes reaccionen a la presión de sus competidores y clientes. Estos resultados son consistentes con los estudios de (AL-Shboul, 2018; Xu et al., 2017).

Con respecto al análisis multigrupo, la mayoría de las relaciones del modelo no presentan diferencias significativas entre los grupos (franquicias y empresas familiares), sin embargo, la relación entre el soporte de alta dirección y la asimilación del ERP es significativamente distinto entre estos tipos de empresa. Conviene resaltar que para las franquicias el soporte de la alta dirección es significativa, mientras que para las empresas familiares no lo es. Esto puede deberse a que el soporte de la alta dirección en la franquicia está estandarizado, ya que tiene un modelo de negocio en el cual la gerencia decide invariablemente cualquier cambio, ya sea en el proceso o en la operación. Otra relación que también resultó significativamente distinta fue la de ventaja relativa y asimilación, mientras que para las empresas familiares es significativa, para las franquicias no es significativa, esta tal vez pueda ser originada porque en las empresas familiares atribuyen la ventaja relativa al sistema ERP, por adopción razonada mientras que en la franquicia es por requerimiento procedimental.

\section{○. Conclusiones.}

El estudio en empresas con gestión divergentes -empresas familiares y franquicias- comprobó que, si bien los sistemas de planificación empresarial, conocidos como ERP, contribuyen al desempeño de las firmas, el efecto es determinado por las características organizacionales con las que la asimilación a su interior define la intensidad y su dirección dados los esfuerzos diferenciados por sus peculiaridades. Asimismo, se obtuvo evidencia de la incidencia de la adopción de estrategias y 
tecnología de información en todas las áreas de gestión y operación de la empresa. Sin embargo, al comparar los factores determinantes del desempeño en empresas cuyos sistemas de control con alta o baja flexibilidad organizacional, la eficacia difiere. El estudio postula un modelo explicativo de 70 franquicias y 102 empresas familiares con la perspectiva teórica del Modelo TOE (TecnologíaOrganización-Ambiente), el cual incorpora la orientación a la organización y tiene en cuenta las Teorías Visión Basada en los Recursos (RBV), Acción Razonada (TAR) y Difusión de las Innovaciones (DOI) complementarias entre sí; de manera que el razonamiento y argumentación fortalece el enfoque de organización encontrándose diferencias en la gestión de franquicias y empresas familiares. Los sistemas empresariales para mejora del desempeño suelen ser conocidos y paulatinamente aceptados. Pese a ello, aún existe desconocimiento o subestimación de la utilidad y beneficios de estos sistemas de planificación. En este sentido, aún no se tiene una fuerte percepción del efecto de la asimilación del ERP como sistema de control gerencial que mejora tanto los rendimientos financieros como no financieros de ambos tipos de empresa, sin embargo, aunque menor, se aprecia un impacto en el desempeño no financiero, esto podría deberse a que resulta más difícil apreciar los beneficios intangibles; al implementar el ERP. El trabajo postulaba que la asimilación del ERP en la organización es vital para lograr el efecto positivo en el desempeño, apoyado con la compatibilidad del sistema, cuando en realidad se encontró que lo limita teniendo en cuenta las características o el tipo de empresa, una razón pudiera ser la falta de integración del sistema con las actividades y cultura organizacional propia.

El presente estudio encuentra un hueco en la literatura revisada al explorar la actuación de la gerencia en la implementación de los sistemas gerenciales. La principal contribución de la investigación es dar a conocer y comprender cuáles son los impulsores tecnológicos, organizacionales y ambientales de la asimilación del ERP, aunque los datos iniciales manifiestan que la adopción de sistemas de gestión empresarial se da por seguir los pasos de los competidores, el análisis evidencia que la transferencia de conocimiento y la presión competitiva son en realidad los propulsores de la asimilación del ERP, más allá de los beneficios esperados. Partiendo de ello, un hecho no esperado fue encontrar que, en el aspecto tecnológico, el ERP supone proveer una ventaja relativa, sin embargo, para la franquicia tuvo un sentido negativo suponiendo así que este tipo de empresa tiene ya una ventaja competitiva en el aspecto gerencial e incorpora el ERP como un sistema tecnológico, mientras que para una empresa familiar resultaría ser su ventaja competitiva.

En cuanto a la complejidad, los resultados muestran un efecto positivo en la asimilación del ERP, esto sugiere que, las empresas restauranteras no ven a la complejidad como un inhibidor para la asimilación del ERP, tal vez porque utilizan solo aquellos módulos necesarios, superando el sistema 
complejo, ante la evidente mejora en la gestión de sus recursos, representando el sistema empresarial probablemente para las franquicias un control y para las empresas familiares eficiencia.

Otro hallazgo inesperado fue el sentido negativo del ajuste organizacional que le implica a una franquicia la adopción de un ERP como sistema tecnológico, por su nivel de estandarización opera coordinadamente la gestión administrativa, donde quizá lo que requiera es el instrumento tecnológico para mejorar su desempeño en ambos sentidos, financiero y no financiero; siendo el primero el de mayor importancia usualmente, pero más para una empresa familiar donde la selección del sistema ERP se basa mayormente en el costo del mismo y no en la adecuación del sistema a los procesos organizativos.

Una implicación importante de la investigación fue la gestión diferenciada que se conjetura en la investigación es la transferencia de conocimiento dada en la asimilación del sistema empresarial; en el caso de las empresas familiares, la transferencia de conocimiento de padres a hijos es un factor clave, el conocimiento del producto o servicio, el conocimiento técnico y el de la gestión de la empresa, resultando un recurso valioso para crear la ventaja competitiva de la empresa familiar y, de esta forma sobrevivir a largo plazo. En el caso de las franquicias, las empresas adquieren los conocimientos de los socios, reforzando y complementando los recursos limitados de la empresa, formalizando a través de la franquicia, el acceso a dichos recursos y capacidades. La experiencia y el compromiso del franquiciador facilita la transferencia de conocimiento al ayudar al franquiciado a adquirir conocimientos relacionados con ventas, marketing y manuales operativos del sistema ERP, entre otros. Si el sistema ERP es una plataforma donde se gestiona la relación con los clientes, la gestión estratégica e incluso la gestión del conocimiento, resulta trascendental que las empresas restauranteras, valoren y no subestimen la utilidad de los sistemas ERP.

Por su parte, la presión competitiva es reconocida como una necesidad estratégica, al cambiar las reglas del juego, modificando la estructura interna de la empresa en sus actividades y operaciones, a través de la asimilación del ERP, enfrentándose de manera más agresiva ante los rivales superiores, en otras palabras, la asimilación del ERP es acelerada por la presión competitiva, teniendo como resultado un mejor desempeño financiero y no financiero. Sin embargo, para que esto último suceda, es preponderante que se tenga un compromiso total de parte de los directivos, para que, mediante la participación activa, la visión y la dirección de los ejecutivos de la alta dirección, con un enfoque de los esfuerzos conjuntos hacia la realización de los beneficios organizacionales, se logre un desempeño integral, es decir un desempeño financiero y no financiero. En lo futuro, la investigación podría basarse en un estudio analizando distintos sectores e industrias, considerando además entrevistas a diferentes partes interesadas, como personal de TI, y con más participantes para mejores tendencias y obtener comparaciones más confiables. 


\section{Bibliografía y referencias documentales}

Abd, M. A., Nasr, E. S., \& Geith, M. H. (2017). Benefits and challenges of cloud ERP systems e A systematic literature review. Future Computing and Informatics Journal, 1, 1-9. https://doi.org/10.1016/j.fcij.2017.03.003

Ahani, A., Rahim, N. Z. A., \& Nilashi, M. (2017). Forecasting Social CRM Adoption in SMEs : A Combined SEM-Neural Network Method. Computers in Human Behavior, 75, 560-578. https://doi.org/10.1016/j.chb.2017.05.032

Al-Qirim, N. (2006). The Role of the Government and E-Commerce Adoption in Small Businesses in New Zealand, 4(4), 293-313.

AL-Shboul. (2018). Towards Better Understanding of Determinants Logistical Factors in SMEs for Cloud ERP Adoption in Developing Economies. Business Process Management. https://doi.org/10.1108/BPMJ-01-2018-0004

Albar, A. M., \& Hoque, M. R. (2017). Factors Affecting Cloud ERP Adoption in Saudi Arabia : An Empirical Study. Information Development, 1-15. https://doi.org/10.1177/0266666917735677

Alshamaila, Y., Papagiannidis, S., \& Li, F. (2013). Cloud Computing Adoption by SMEs in the North East of England A Multi-Perspective Framework. Journal of Enterprise Information Management, 26(3), 250275. https://doi.org/10.1108/17410391311325225

Argote, L., \& Ingram, P. (2000). Knowledge transfer: A basis for competitive advantage in firms. Organizational Behavior and Human Decision Processes, 82(1), 150-169. https://doi.org/10.1006/obhd.2000.2893

Awa, H., Baridam, D., \& Nwibere, M. (2015). Demographic Determinants of Electronic Commerce (EC) Adoption by SMEs. Journal of Enterprise Information Management, 28(3), 326-345.

Awa, H. O., \& Kalu, S. E. (2010). Repositioning the Non-incremental Changes and Business Strategic Windows Correlates. International Journal of Business and Management, 5(2), 184-193.

Awa, H. O., \& Ojiabo, O. (2016). A Model of Adoption Determinants of ERP within T-O-E Framework. Information Technology \& People, 29(4), 901-930. https://doi.org/10.1108/ITP-03-2015-0068

Awa, H., Ojiabo, O., \& Orokor, L. (2017). Integrated Technology-Organization-Environment ( T-O-E ) Taxonomies for Technology Adoption. Journal of Enterprise Information, 30(6), 893-921. https://doi.org/10.1108/JEIM-03-2016-0079

Balaid, A., Abd Rozan, M., \& Abdullah, S. N. (2017). Conceptual Model for Examining Knowledge Maps Adoption in Software Development Organizations. Asian Social Science, 10(15), 118-132. https://doi.org/10.5539/ass.v10n15p118

Barroso, A., Sanguino, R., \& Bañegil, T. (2016). An Empirical Study About Knowledge Transfer, Entrepreneurial Orientation and Performance in Family Firms. European J. of International Management, 10(5), 534-557. https://doi.org/10.1504/EJIM.2016.078790

Baykasoğlu, A., \& Gölcük, İ. (2017). Development of a two-phase structural model for evaluating ERP critical success factors along with a case study. Computers \& Industrial Engineering, 106, 256-274. https://doi.org/10.1016/j.cie.2017.02.015

Borgman, H. P., Bahli, B., Heier, H., \& Schewski, F. (2013). Cloudrise : Exploring Cloud Computing Adoption and Governance With the TOE Framework. In 46th Hawaii International Conference on Systems Sciences (pp. 4425-4435). https://doi.org/10.1109/HICSS.2013.132

Bose, R., \& Luo, X. (2011). Integrative Framework for Assessing Firms ' Potential to Undertake Green IT Initiatives Via Virtualization - A Theoretical Perspective. Journal of Strategic Information Systems, 20(1), 38-54. https://doi.org/10.1016/j.jsis.2011.01.003

Bradford, M., \& Florin, J. (2003). Examining the Role of Innovation Diffusion Factors on the Implementation Success of Enterprise Resource Planning Systems. Accounting Information Systems, 4, 205-225. https://doi.org/10.1016/S1467-0895(03)00026-5

Bruhn, M., Karlan, D. S., \& Schoar, A. (2012). The Impact of Consulting Services on Small and Medium Enterprises: Evidence from a Randomized Trial in Mexico. Journal of Political Economy, 126(2), 635687. https://doi.org/10.2139/ssrn.2010710

Cahyadi, I. (2019). A Combined ANP, TOPSIS and MCGP Approach to Select Knowledge Transfer Strategy: A Case Study in Indonesian SMEs ERP System Implementation A Combined ANP, TOPSIS and MCGP Approach to Select Knowledge Transfer Strategy : A Case Study in Indonesian SMEs E. Series, 
I O P Conference Science, Materials. https://doi.org/10.1088/1757-899X/505/1/012001

Chang, M. K., Cheung, W., Cheng, C. H., \& Yeung, J. H. Y. (2008). Understanding ERP System Adoption from the User's Perspective. International Journal of Production Economics, 113(2), 928-942. https://doi.org/10.1016/j.ijpe.2007.08.011

Chang, S.-I., Hung, S.-Y., \& Yen, D. (2010). Critical Factors of ERP Adoption for Small- and Medium- Sized Enterprises : An Empirical Study. Journal of Global Information Management, 18(3), 82-106. https://doi.org/10.4018/jgim.2010070104

Chatterjee, D., Grewal, R., \& Sambamurthy, V. (2002). Shaping up for E-Commerce: institutional Enablers of the Organizational Assimilation of Web Technologies. MIS Quarterly, 26(2), 65-89.

Chin, W. (1998). Issues and Opinion on Structural Equation Modeling. MIS Quarterly, 22(1).

Cohen, J. (1992). A Power Primer. Psychological Bulletin, 112(1), 155-159.

Darr, E. D., Argote, L., \& Epple, D. (1995). The Acquisition , Transfer , and Depreciation of Knowledge in Service Organizations : Productivity in Franchises. Management Science, 41(11), 1750-1762.

Darr, E. D., \& Kurtzberg, T. R. (2000). An Investigation of Partner Similarity Dimensions on Knowledge Transfer. Organizational Behavior and Human Decision Processes, 82(1), $28-44$. https://doi.org/10.1006/obhd.2000.2885

Davenport, T., \& Prusak, L. (2000). Working Knowledge : How Organizations Manage What They Know. Harvard Business Press, 1-15.

Delone, W. H., \& Mclean, E. R. (1992). Information Systems Success : The Quest for the Dependent Variable, (August 2015).

Er, M., Zayin, S. O., \& Pamungkas, F. J. (2017). ERP Post Implementation Review with Process Mining : A Case of Procurement Process. Procedia Computer Science, 124, 216-223. https://doi.org/10.1016/j.procs.2017.12.149

Eze, S. C., Awa, H. O., Okoye, J. C., Emecheta, B. C., \& Anazodo, R. O. (2013). Determinant Factors of Information Communication Technology ( ICT ) Adoption by Government-Owned Universities in Nigeria: a Qualitative Aproach. Journal of Enterprise Information Management, 26(4), 427-443. https://doi.org/10.1108/JEIM-05-2013-0024

Fichman, R. G., \& Kemerer, C. F. (2016). The Illusory Diffusion of Innovation : An Examination of Assimilation Gaps, 10(3), 255-275.

Filius, R., de Jong, J., \& Roelofs, E. C. (2000). Knowledge management in the HRD office : a comparison of three cases. Journal of Workplace Learning, 12(7), 286-295.

Fishbein, M., \& Azjen, I. (1975). Belief, Attitude, Intention and Behavior: An Introduction to theory and research. Reading, MA: Addison-Wesley. Retrieved from people.umass.edu/aizen/f\&a1975.html\%0D

Gangwar, H., Date, H., \& Ramaswamy, R. (2015). Understanding determinants of cloud computing adoption using an integrated TAM-TOE model. Journal of Enterprise Information Management, 28(1), 107-130. https://doi.org/http://dx.doi.org/10.1108/MRR-09-2015-0216

Gangwar, H., Date, H., \& Raoot, A. D. (2014). Review on IT Adoption : Insights from Recent Technologies. Journal of Enterprise Information Management, 27(4), 488-502. https://doi.org/10.1108/JEIM-082012-0047

Garg, P., \& Chauhan, A. (2015). Factors affecting the ERP implementation in Indian retail sector, 22(7), $1315-1340$.

Ghobakhloo, M., Arias-Aranda, D., \& Benitez-Amado, J. (2011). Adoption of E-Commerce Applications in SMEs. Industrial Management \& Data Systems, 3(8), 1238-1269. https://doi.org/10.1108/02635571111170785

Ghobakhloo, M., Azar, A., \& Tang, S. H. (2018). Business value of enterprise resource planning spending and scope. https://doi.org/10.1108/K-01-2018-0025

Govindaraju, R., Hariadi, R. A. ., \& Sidiq, A. Z. (2015). ERP Assimilation and Benefit Realization : Analyzing the Influence of Leader Characteristics. International Conference on Technology Systems and Innovation (ICITSI), 16(19), 16-19.

Govindaraju, R., Salajar, R. T., Chandra, D. R., \& Sudirman, I. (2015). Acceptance and Usage of ERP Systems : The Role of Institutional Factors in ERP. Proceedings of the IEEE IEEM, 1292-1296.

Goyette, S., Cassivi, L., Courchesne, M., \& Elia, E. (2015). The ERP Post- implementation Stage: a Knowledge Transfer Challenge. International Journal of Information Systems and Project Management, $3(2), 5-19$.

Greenhalgh, T., Macfarlane, G., Bate, P., \& Kyriakidou, O. (2004). Diffusion of Innovations in Service Organizations: Systematic Review and Recommendations. The MilbankQuarterly, 82(4), 581-629. 
Hair, J. F., Ringle, C. M., \& Sarstedt, M. (2013). Partial Least Squares Structural Equation Modeling: Rigorous Applications, Better Results and Higher Acceptance. Long Range Planning, 46(1-2), 1-12. https://doi.org/10.1016/j.lrp.2013.01.001

Hair, J. F., Risher, J. J., Sarstedt, M., \& Ringle, C. M. (2019). When to use and how to report the results of PLS-SEM. European Business Review, 31(1), 2-24. https://doi.org/10.1108/ebr-11-2018-0203

Hair, J. J. F., Anderson, R. E., Tatham, R. L., \& Black, W. C. (2007). Análisis Multivariante. España: Pearson Prentice Hall International, Inc.

Hair, J. J. F., Hult, G. T., Ringle, C. M., Sarstedt, M., Castillo, J., Cepeda, G. A., \& Roldán, J. L. (2019). Manual de Partial Least Squares Structural Equation Modeling (PLS-SEM). España: OmniaScience. https://doi.org/https://doi.org/10.3926/oss.37

Hair, J. J. F., Hult, G. T., Ringle, C., \& Sarstedt, M. (2017). A Primer on Partial Least Squares Structural Equation Modeling (PLS-SEM) (2nd Editio). Sage Publications.

Hair, J. J., Hult, G. T., Ringle, C. M., \& Sarstedt, M. (2017). A Primer on Partial Least Squares Structural Equation Modeling (PLS-SEM) (Second Edi).

Hair, J., Ringle, C., \& Sarstedt, M. (2011). PLS-SEM: Indeed and Silver Bullet. Journal of Marketing Theory and Practice, 19(2), 139-151. Retrieved from https://www.tandfonline.com/doi/abs/10.2753/MTP10696679190202

Henseler, J. (2017). Partial Least Squares Path Modeling. Advanced Methods for Modeling Markets, 361381. https://doi.org/10.1007/978-3-319-53469-5

Henseler, J., \& Chin, W. W. (2010). A comparison of approaches for the analysis of interaction effects between latent variables using partial least squares path modeling. Structural Equation Modeling, 17(1), 82-109. https://doi.org/10.1080/10705510903439003

Henseler, J., Hubona, G., \& Ray, P. A. (2016). Using PLS path modeling in new technology research : updated guidelines, 116(1), 2-20. https://doi.org/10.1108/IMDS-09-2015-0382

Henseler, J., Ringle, C. M., \& Sarstedt, M. (2016). Testing Measurement Invariance of Composites Using Partial Least Squares. International Marketing Review, 33(3), 405-431. https://doi.org/10.1108/IMR09-2014-0304

Henseler, J., Ringle, C. M., \& Sinkovics, R. R. (2009). The Use of Partial Least Squares Path Modeling in International Marketing, 20, 277-319. https://doi.org/10.1108/S1474-7979(2009)0000020014

Huang, T., \& Yasuda, K. (2016). Comprehensive review of Literature Survey Articles on ERP. Business Process Management Journal, 22(1), 2-32.

Hung, W. H., Ho, C. F., Jou, J. J., \& Kung, K. H. (2012). Relationship bonding for a better knowledge transfer climate: An ERP implementation research. Decision Support Systems, 52(2), 406-414. https://doi.org/10.1016/j.dss.2011.09.007

Ifinedo, P. (2011). An Empirical Analysis of Factors nfluencing Internet/E-Business Technologies Adoption by SMEs in Canada. World Scientific, 10(4), 731-766. https://doi.org/10.1142/S0219622011004543

Inkpen, A. C., \& Tsang, E. W. K. (2005). Social Capital Networks, and Knowledge Transfer, 30(1), 146-165.

Instituto Nacional de Estadística y Geografía (INEGI). (2018). Banco de Información Económica BIE. https://www.inegi.org.mx/sistemas/bie/?idserPadre $=10200043002000200118$

Instituto Nacional de Estadística y Geografía (INEGI). (2019, dic). Encuesta Nacional de Ocupación y Empleo Trimestral. https://www.inegi.org.mx/sistemas/Infoenoe/Default_15mas.aspx

Jeyaraj, A., Rottman, J. W., \& Lacity, M. C. (2006). A Review of the Predictors, Linkages, and Biases in IT Innovation Adoption Research. Journal of Information Technology, 21, 1-23. https://doi.org/10.1057/palgrave.jit.2000056

Kannabiran, G., \& Dharmalingam, P. (2012). Enablers and Inhibitors of Advanced Information Technologies Adoption by SMEs An Empirical Study of Auto Ancillaries in India. Journal of Enterprise Information Management, 25(2), 186-209. https://doi.org/10.1108/17410391211204419

Kiriwandeniya, I., Ruwan, V. U. A., Samarasinghe, S. S. U., Samarakoon, S. M. P. A., Kahandawarachchi, C., \& Thelijjagoda, S. (2013). Post Implementation Framework for ERP Systems with Special Reference to Sri Lanka. In The 8th International Conference on Computer Science \& Education (pp. 508-513).

Kouki, R., Poulin, D., \& Pellerin, R. (2010). The Impact of Contextual Factors on ERP Assimilation : Exploratory Findings from a Developed and a Developing Country. Journal of Global Information Technology Mnagement, 13(1), 28-55. https://doi.org/10.1080/1097198X.2010.10856508

Law, C. C. H., \& Ngai, E. W. T. (2007). ERP systems adoption: An exploratory study of the organizational factors and impacts of ERP success. Information and Management, 44(4), 418-432. 
https://doi.org/10.1016/j.im.2007.03.004

Lee, S. M., Lee, Z., \& Lee, J. (2007). Knowledge transfer in work practice: Adoption and use of integrated information systems. Industrial Management and Data Systems, 107(4), 501-518. https://doi.org/10.1108/02635570710740661

Lee, Z., \& Lee, J. (2000). An ERP Implementation Case Study from a Knowledge Transfer Perspective. Journal of Information Technology, 15, 281-288. https://doi.org/10.1080/02683960010009060

Leyh, C. (2014). Critical Success Factors for ERP Projects in Small and Medium-sized Enterprises - The Perspective of Selected German SMEs. In Proceedings of the 2014 Federated Conference on Computer Science and Information Systems (Vol. 2, pp. 1181-1190). https://doi.org/10.15439/2014F243

Liang, H., Saraf, N., Hu, Q., \& Xue, Y. (2007). Assimilation of Enterprise Systems: The effect of Institutional Pressures and the Mediating Role of Top Management. MIS Quarterly, 31(1), 59-87.

Low, C., Chen, Y., \& Wu, M. (2011). Understanding the determinants of cloud computing adoption. Industrial Management and Data Systems, 111(7), 1006-1023. https://doi.org/10.1108/02635571111161262

Low, K. Y. J., \& Robins, J. A. (2014). Finding knowledge: The role of reputation in knowledge-transfer to Chinese companies. Long Range Planning, 47(6), 353-364. https://doi.org/10.1016/j.lrp.2014.10.001

Maas, J., Van Fenema, P., \& Soeters, J. (2018). Post-Implementation ERP Usage : A Longitudinal Study of the Impact of Control and Empowerment. Information Systems Management, 35(4), 330-347. https://doi.org/10.1080/10580530.2018.1503804

Maguire, S., Koh, S. C. L., \& Magrys, A. (2007). The Adoption of e-business and Knowledge Management in SMEs. Benchmarking: An International Journal, 14(1), 37-58. https://doi.org/10.1108/14635770710730928

Mangula, I., Weerd, I. Van De, \& Brinkkemper, S. (2014). The Adoption of Software-as-a-Service : An Indonesian Case Study. PACIS 2014.

Markus, M. L. (2001). Toward A Theory of Knowledge Reuse : Types of Knowledge Reuse Situations and Factors in Reuse Success, 18(1), 57-93.

Melville, N., Kraemer, K., Gurbaxani, V., Ew, M., \& Kraemer, K. (2004). Information Technology and Organizational Performance: an Integrative Model of IT Business Value. MIS Quarterly, 28(2), 283 322.

Mendoza, S., Mendoza, J., \& Guzmán, E. (2018). E-commerce as a complementary tool in the PYMES in Mexico, 18(2), 245-273.

Morton, N. A., \& Hu, Q. (2008). Implications of the fit between organizational structure and ERP: A structural contingency theory perspective. International Journal of Information Management, 28(5), 391-402. https://doi.org/10.1016/j.ijinfomgt.2008.01.008

Murphy, K. E., \& Simon, S. J. (2002). Intangible benefits valuation in ERP projects, 301-320.

Nandi, M. . L., \& Vakkayil, J. (2018). Absortive Capacity and ERP Assimilation: the Influence of Company Ownership. Business Process Management, 24(3), 695-715.

Ociepa-Kubicka, A. (2017). Advantages of using enterprise resource planning systems ( ERP) in the management process. World Scientific News, 89, 237-243.

Pennings, J. M., \& Harianto, F. (1992). Technological Networking and Innovation Implementation. Organization Science, 3(3), 356-382.

Peppard, J. (2016). A Tool for Balancing Your Company's Digital Investments. Harvard Business Review. Retrieved from https://hbr.org/2016/10/a-tool-for-balancing-your-companys-digital-investments\%0D

Phaphoom, N., Saelee, W., Somjaitaweeporn, T., Yuenyong, S., \& Qu, J. (2018). A Combined Method for Analysing Critical Success Factors on ERP Implementation. 2018 15th International Joint Conference on Computer Science and Software Engineering (JCSSE), 1-6.

Picoto, W. N., Bélanger, F., \& Palma-dos-Reis, A. (2014). An organizational perspective on m-business : usage factors and value determination $\uparrow$. European Journal of Information Systems, 23, 571-592. https://doi.org/10.1057/ejis.2014.15

Podsakoff, P. M., Mackenzie, S. B., Lee, J., \& Podsakoff, N. P. (2003). Common Method Biases in Behavioral Research : A Critical Review of the Literature and Recommended Remedies. Journal Applied Psychology, 88(5), 879-903. https://doi.org/10.1037/0021-9010.88.5.879

Poston, R., \& Grabski, S. (2001). Financial Impacts of Enterprise Resource Planning Implementations. International Journal of Accounting Information Systems, 2(4), 271-294.

Pudjianto, B., Zo, H., Ciganek, A. P., \& Rho, J. J. (2011). Determinants of E-Government Assimilation in Indonesia : An Empirical Investigation Using a TOE Framework. Asia Pacific Journal of Information Systems, 21(1), 49-80. 
Purvis, R. L., Sambamurthy, V., \& Zmud, R. W. (2001). The Assimilation of Knowledge Platforms in Organizations : An Empirical Investigation. Organization Science, 12(2), 117-135.

Reagans, R., Singh, P., \& Krishnan. (2015). Forgotten Third Parties : Analyzing the Contingent Association Between Unshared Third Parties, Knowledge Overlap, and Knowledge Transfer Relationships with Outsiders. Organization Science, 1-15.

Ringle, C. M., Wende, S., \& Becker, J. M. (2015). Smart PLS 3. Boenningstedt: Smart PLS GmbH. Retrieved from http://www.smartpls.com

Rogers, E. M. (1995). Diffussion of Innovation.

Ruivo, P., Oliveira, T., Johansson, B., \& Neto, M. (2013). Differential Effects on ERP Post-Adoption Stages across Scandinavian and Iberian SMEs. Journal of Global Information Management. https://doi.org/10.4018/jgim.2013070101

Ruivo, P., Oliveira, T., \& Neto, M. (2014). Examine ERP post-implementation stages of use and value: Empirical evidence from Portuguese SMEs. International Journal of Accounting Information Systems. https://doi.org/10.1016/j.accinf.2014.01.002

Ruivo, P., Oliveira, T., \& Neto, M. (2015). Using resource-based view theory to assess the value of ERP commercial-packages in SMEs. Computers in Industry, 73, 105-116. https://doi.org/10.1016/j.compind.2015.06.001

Sadrzadehrafiei, S., Chofreh, A. G., Hosseini, N. K., \& Sulainman, R. (2013). The Benefits of Enterprise Resource Planning ( ERP) System Implementation in Dry Food Packaging Industry. Procedia Technology, 11, 220-226. https://doi.org/10.1016/j.protcy.2013.12.184

Sarstedt, M., Henseler, J., \& Ringle, C. (2011). Multi-Group Analysis in Partial Least Squares ( PLS ) Path Modeling : Alternative Methods and Empirical Results, 22, 195-218. https://doi.org/10.1108/S14747979(2011)0000022012

Segars, A. H. (1997). Assessing the Unidimensionality of Measurement : a Paradigm and Illustration Within the Context of Information Systems, 25(1), 107-121.

To, M. L., \& Ngai, E. W. T. (2006). Predicting the organisational adoption of B2C e-commerce: An empirical study. Industrial Management and Data Systems, 106(8), 1133-1147. https://doi.org/10.1108/02635570610710791

Tornatzky, L., \& Fleischer, M. (1990). The Processes of Technological Innovation. Lexington, MA: Lexington Books.

Vaghefi, I., Lapointe, L., \& Shahbaznezhad, H. (2018). A multilevel process view of organizational knowledge transfer: enablers versus barriers. Journal of Management Analytics, 5(1), 1-17. https://doi.org/10.1080/23270012.2018.1424572

Vluggen, M. (2005). The Determinants of Enterprise Resource Planning ( ERP ) Systems Usage Levels : An Empirical Study. The 5th European Conference on Accounting Information Systems, Copenhagen, Denmark.

Volkoff, O., Elmes, M. B., \& Strong, D. M. (2004). Enterprise Systems , Knowledge Transfer and Power Users. The Journal of Strategic Information Systems, 13(4), 279-304. https://doi.org/10.1016/j.jsis.2004.11.004

Wang, E. T. G., Shih, S.-P., Jiang, J. J., \& Klein, G. (2008). The Consistency Among Facilitating Factors and ERP Implementation Success : A Holistic View of Fit. The Journal of Systems and Software, 81, 16091621. https://doi.org/10.1016/j.jss.2007.11.722

Wang, Y.-S., Li, H.-T., Li, C.-R., \& Zhang, D.-Z. (2016). Factors Affecting Hotels 'Adoption of Mobile Reservation Systems : A Technology-Organization-Environment Framework. Tourism Management, 53, 163-172. https://doi.org/10.1016/j.tourman.2015.09.021

$\mathrm{Xu}, \mathrm{W} ., \mathrm{Ou}, \mathrm{P} ., \mathrm{\&}$ Fan, W. (2017). Antecedents of ERP assimilation and its impact on ERP value : A TOEbased model and empirical test. Information Systems Frontiers, 19, 13-30. https://doi.org/10.1007/s10796-015-9583-0

Yoon, T. E., \& George, J. F. (2013). Why aren 't Organizations Adopting Virtual Worlds ? Computers in Human Behavior, 29, 772-790. https://doi.org/10.1016/j.chb.2012.12.003

Zhu, K., \& Kraemer, K. L. (2005). Post-adoption variations in usage and value of e-business by organizations: Cross-country evidence from the retail industry. Information Systems Research, 16(1), 61-84. https://doi.org/10.1287/isre.1050.0045 
Zhu, K., Kraemer, K. L., Xu, S., \& Dedrick, J. (2004). Information technology payoff in E-Business environments: An international perspective on value creation of E-Business in the financial services industry. Journal of Management Information Systems, 21(1), 17-54. https://doi.org/10.1080/07421222.2004.11045797

Zhu, Y., Li, Y., Wang, W., \& Chen, J. (2010). Whats Leads to Post-Implementation Success of ERP? An Empirical Study of the Chinese Retail Industry. International Journal of Information Management, 30(3), 265-276. 


\section{Números anteriores}

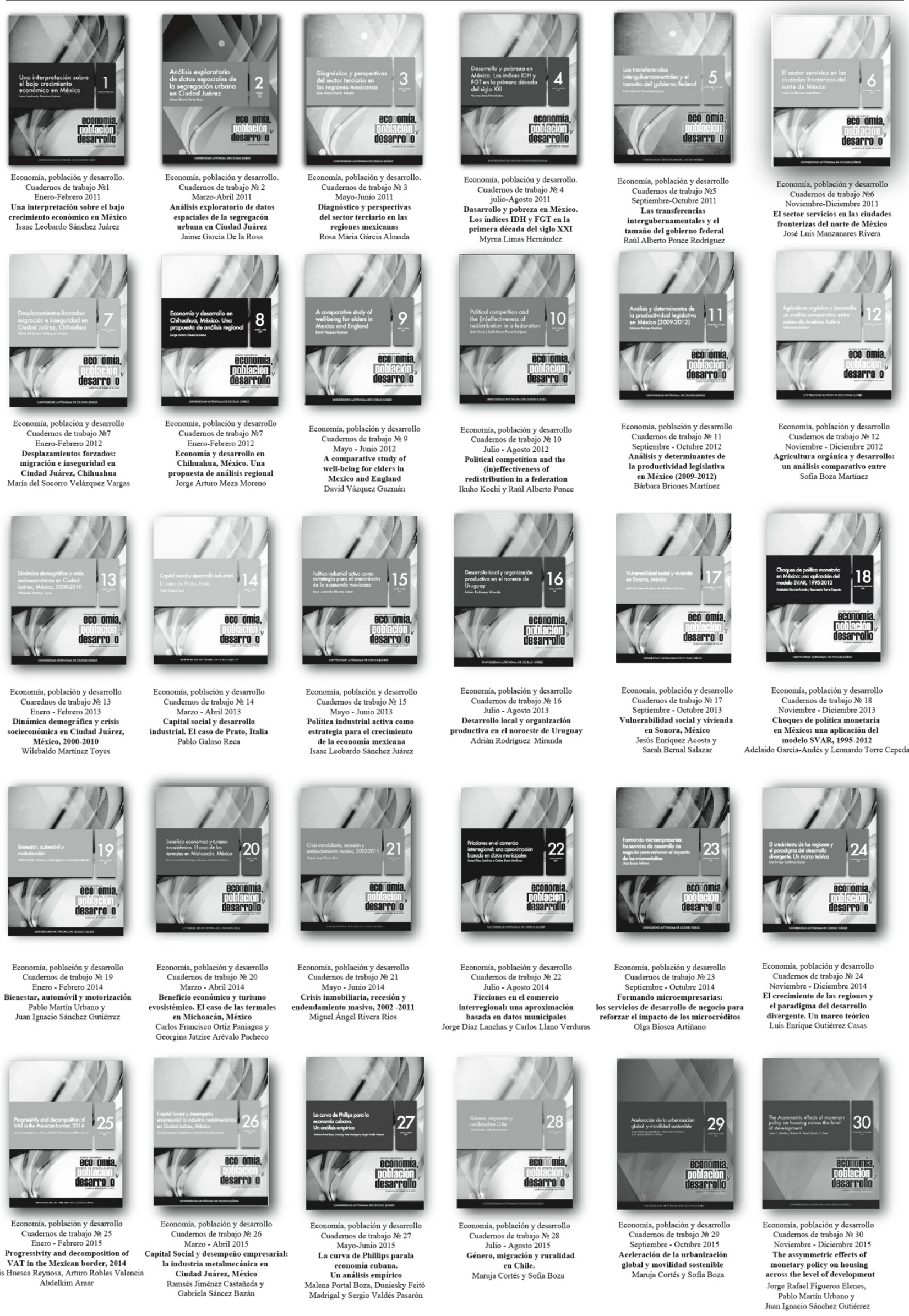



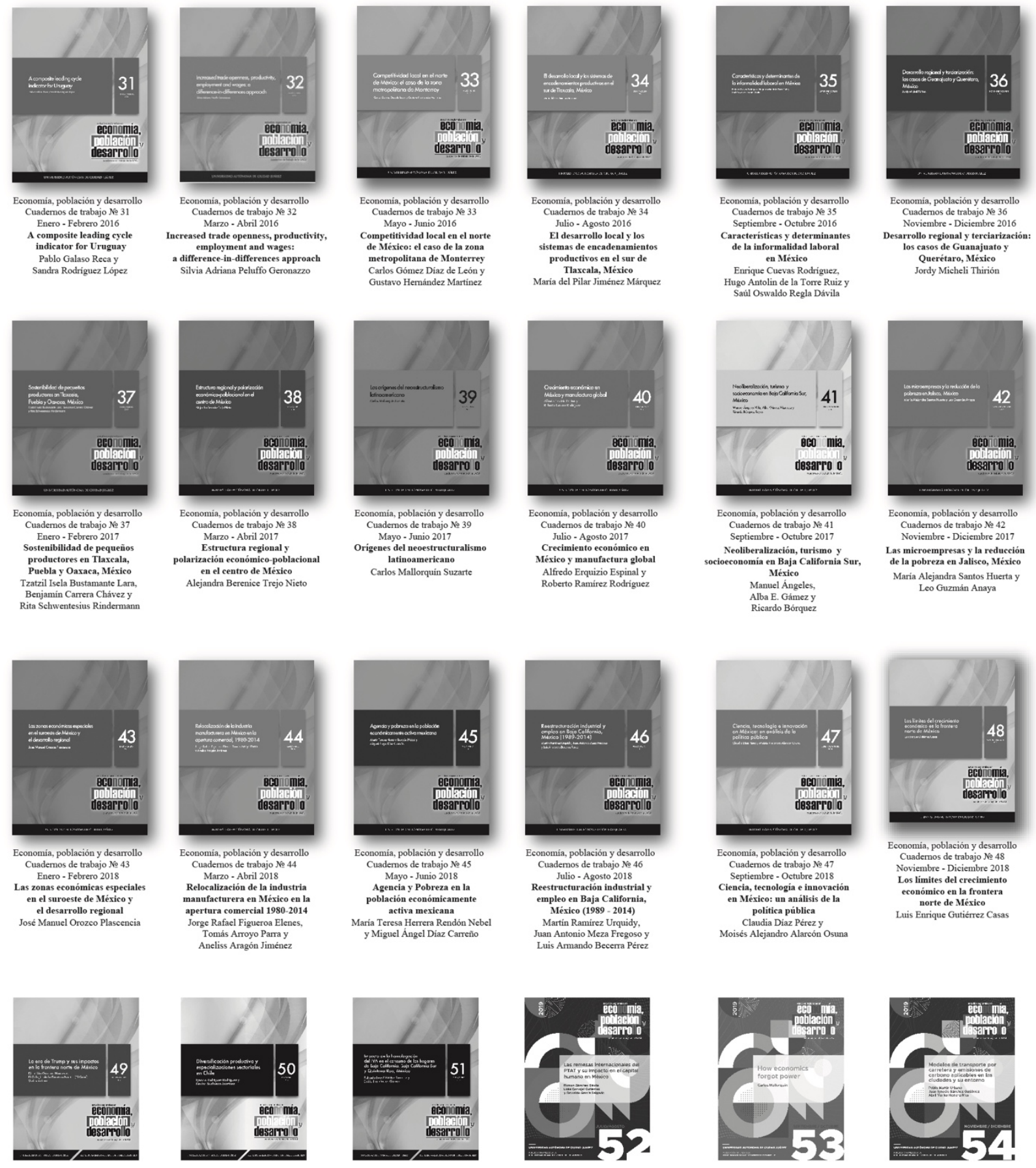

Economia, población y desararollo
Cuadernos de trabajo No 49
Enero - Febrero 2019

La era de Trump y sus impactos
en la frontera norte de Mérico

Dirección General doroeste

Varios autores

Economia, población y desarrollo
Cuadernos de trabajo № 50 Marzo - Abril 2019

Diversificación productiva y
especializaciones sectoriales en Chile

Economia, población y desarrollo
Cuadernos de trabajo № 51 Mayo - Junio 2019
Impacto de la homologación del IVA
en el consumo de los hogares de en el consumo de los hogares de
Baja Califoria, Baja Califoria Sur Ignacio Rodriguez Rodriguez
Paulina Sanhueza Martinez
Economiá, población y desarrollo
Cuadernos de trabajo № 52 gosto 2019 Las remesas internacionales
del PTAT y su impacto en el capital humano
ent Romin Sánchez Dávila Lidia Carvajal Gutiérrezy
Oswaldo Garcia Salgado Rolando Israel Valdez Ramirez y
Emilio Hermaindez Gómez
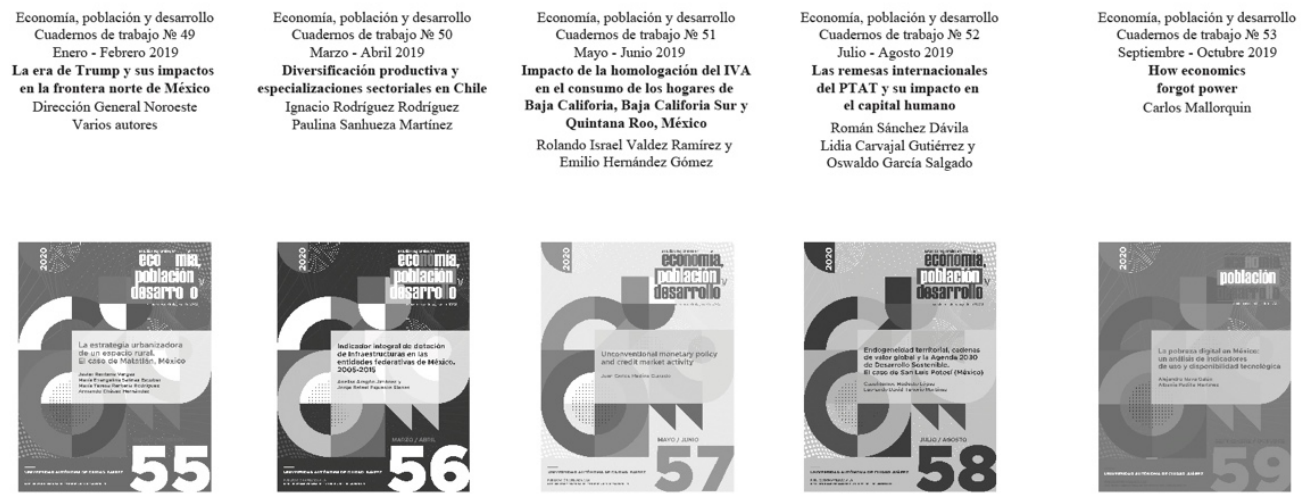

Economia, población y desarrollo
Cuadernos de trabajo № 54 Noviembre - Diciembre 2019
Modelos de transporte por carretera y Modelos de transporte por carretera y
emisiones de carbono aplicables en las ciudades y su entorno Pablo Martin Urbano,
Juan Ignacio Sánchez Gutierrez
y Abril Yuriko Herrera Rios

Economia, población y desarrollo Enero - Febrero 2020 La estrategia urbanizadora
de un espacio rural. de un espacio rural.
El caso de Matatlán, México Javier Renteria Vargas, Maria Evangelina Salinas Escobar,
Maria Teresa Renteria Rodriguez Armando Chávez Hernánde

Economia, población y desarrollo
Cuadermos de trabajo No 56 Cuademos de trabajo o
Marzo-Abril 2020 Indicador integral de dotación de
infraestructuras en las entidades Tederativas a 2015 Aneliss Aragón Jiménez y
Jorge Rafael Figueroa Elenes
Economia, población y desarrollo
Cuadernos de trabaje No 57 Mayo - Junio 2020

Unconventional monetary policy
and creditmarket activity Juan Carlos Medina Guirado
Economia, población y desarrollo Cuadernos de trabajo.
Julio - Agosto 2020 Endogeneidad territorial, cadenas de valor global y la
Agenda 2030 de Desarrollo Sostenible. Agenda 2030 de Desarrollo Sostenible Cuauhtémoc Modesto López y
Leonardo David Tenorio Martinez
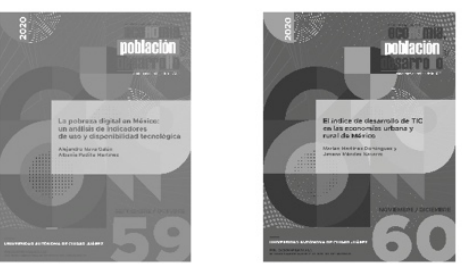

Economia, población y desarrollo Septiembre - Octubre 2020 La pobreza digital en México: disponibilidad tecnológica Alejandro Nava Galán y
Economia, población y desarrollo de trabajo № El indice de desarrollo de TIC en las economias
urbana y rural de México Marlen Martinez Domingue zy 
Números anteriores

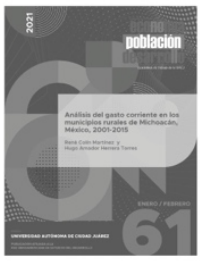

Economía, población y desarrollo
Cuadernos de trabajo No $_{0}$ Enero - Febrero 2021

Anuicipies rurales de Michoente

México, 2001-2015

René Colín Martínez y
Hugo Amador Herrera Torre

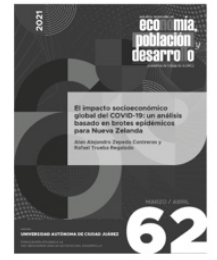

Economia, población y desarrollo Cuadernos de trabajo № 62 El imarzo - Abril 2021 El impacto sociocenómimico
global del COVID-19: nanálisis basadoe brotes epidémicos par
Nueva Zelanda Alan Alejandro Zepeda Contreras y
Rafael Trueba Regalado

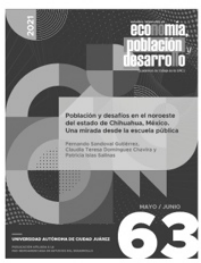

Economiá, población y desarrollo Cuadernos de trabajo $\mathrm{N}_{2} 63$
Mayo - Junio 2021 Población y desafios en el noroess
del estado de Chituahua, Mexice.

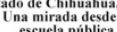

Fernando Sandoval Gutiérrez,
Claudia Teresa Dominguez Chavi y Patricia Islas Salinas

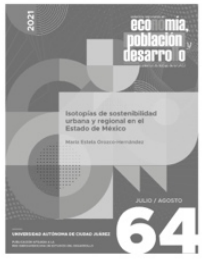
Economiá, población y desarrollo
Cuadernos de trabajoj $\mathrm{Ne}_{2} 64$ Jutio - Agosto 2021 it Isotopias de sostenibilidad
urbana y regional
en el Estado de México Maria Estela Orozco-Hernández 


\section{Para el documento general:}

Tipo de letra: Times New Roman.

Tamaño: 11 puntos.

Interlineado: 1.5 espacios.

Títulos y subtítulos:

El texto principal en 11 puntos. Títulos 12 puntos (en resaltado). Subtítulos 11 puntos. Cada título y subtítulo deberá numerarse bajo el siguiente orden: 1, 1.1, 2, 2.1, 2.2...

La extensión máxima de los cuadernos de trabajo será de 40 cuartillas.

La primera vez que se emplee una sigla en el texto se especificará primero su equivalencia completa y después la sigla.

\section{Hoja de presentación:}

Título:

14 puntos, centrado, resaltado.

Nombre de autor(es):

12 puntos

Resumen y abstract:

Debe incluir resumen en español y abstract (diez puntos), no mayor a 250 palabras

Palabras clave:

Incluir entre tres y cinco palabras clave, en español e inglés

Referencia del autor o autores:

Institución de adscripción, grado académico y líneas-grupos de investigación que desarrolla y a los que pertenece.

\section{Sistema de referencia de citas:}

Harvard-APA

Las citas bibliográficas en el texto deberán incluir entre paréntesis sólo el apellido del autor, la fecha de publicación y el número de página; por ejemplo: (Quilodrán, 2001: 33).

\section{Notación en sección de bibliografía y fuentes de información:}

Se deberá incluir al final del texto. Toda referencia deberá estar mencionada en el texto o notas de pie de página.

Cada referencia iniciará con el primer apellido o los apellidos, luego el nombre del autor, y después, entre paréntesis, el año de publicación seguido de un punto. Ejemplos:

Se deberá incluir al final del texto. Toda referencia deberá estar mencionada en el texto o notas de pie de página.

Cada referencia iniciará con el primer apellido o los apellidos, luego el nombre del autor, y después, entre paréntesis, el año de publicación seguido de un punto. Ejemplos: 
Artículo:

Ros, Jaime (2008). "La desaceleración del crecimiento económico en México desde 1982", en Trimestre Económico, vol. 75, núm. 299, pp. 537-560.

Libro:

Villarreal, René (2005). Industrialización, competitividad y desequilibrio externo en México.

Un enfoque macroindustrial y financiero (1929-2010), México, Fondo de Cultura Económica.

Capítulo de libro:

Castillo, Manuel Ángel (2003). "La política de inmigración en México: un breve recuento", en Manuel Ángel Castillo, Alfredo Lattes y Jorge Santibáñez (coords.), Migración y fronteras, Tijuana,

El Colegio de la Frontera Norte / Asociación Latinoamericana de Sociología / El Colegio de México, pp. 425-451.

\section{Notas de pie de página:}

Se utilizarán para hacer indicaciones complementarias, aclaraciones o ampliación de una explicación. La nota de pie de página en Times New Roman, 10 puntos.

\section{Tipología de imágenes dentro del texto:}

Cuadro

Gráfica

Diagrama

Mapa

Figura

Todas las imágenes deben ser numeradas y mencionadas dentro del texto. A toda imagen debe incluirse la fuente.

Las indicaciones de la imagen: tipo y número de imagen, título de imagen y fuente se escriben en 10 puntos. En el texto poner como imagen los mapas, figuras, gráficas y diagramas -con el ánimo de no perder el formato realizado por el autor.

\section{Ecuaciones y fórmulas:}

Si se utilizan ecuaciones o fórmulas deberá utilizarse el editor de ecuaciones de Word y numerarse.

\section{Envío de trabajos}

Los trabajos deben ser enviados a la dirección de correo: lgtz@uacj.mx. Con el Dr. Luis Enrique Gutiérrez Casas, editor de esta publicación.

La aceptación de cada colaboración dependerá de la evaluación de dos dictaminadores especialistas en la materia que se conservarán en el anonimato, al igual que el autor (autores) para efectos de la misma. 


\section{For General Document:}

Font type: Times New Roman.

Size: font size 11.

Paragraph: 1.5 line spacing.

Titles and subtitles: Main text font size 11. Titles font size 12 (Bold). Subtitles font size 11.

Each title and subtitle should be numbered in the following order: 1, 1.1, 2, 2.1, 2.2...

The maximum length of the workbooks will be 40 pages.

The first time an abbreviation is used in the text will be specified first complete equivalence and then stands.

\section{Front cover:}

Title:

Font size 14, centered, Bold.

Author name(s):

Font size 12.

Abstract:

It should include abstract in Spanish and abstract (font size 10), no more than 250 words.

Keywords:

Include three to five keywords, in Spanish and English.

Reference of author:

Institution of affiliation, academic degree and line-developed by research groups and belonging.

\section{Bibliographical appointment system:}

Harvard-APA

Citations in the text should include between parentheses only the author's name, publication date and page number, for example:

(Quilodrán, 2001: 33).

\section{Notation about Bibliography section and Information fonts:}

Should be included at the end of the text. All references must be mentioned in the text or footnotes page.

Each reference starts with the first name or last name, then the name of the author, and then, in parentheses, the year of publication followed by a period. Examples:

Article:

Ros, Jaime (2008). "La desaceleración del crecimiento económico en México desde 1982", en Trimestre Económico, vol. 75, núm. 299, pp. 537-560. 


\section{Editorial Guidelines}

Book:

Villarreal, René (2005). Industrialización, competitividad y desequilibrio externo en México. Un enfoque macroindustrial y financiero (1929-2010), México, Fondo de Cultura Económica.

Book chapter:

Castillo, Manuel Ángel (2003). "La política de inmigración en México: un breve recuento", en Manuel Ángel Castillo, Alfredo Lattes y Jorge Santibáñez (coords.), Migración y fronteras, Tijuana, El Colegio de la Frontera Norte / Asociación Latinoamericana de Sociología / El Colegio de México, pp. 425-451.

\section{Footnotes:}

Must be used to make additional indications, clarification or expansion of an explanation. The footnotes must be in Times New Roman, font size 10 .

\section{Image typology inside text:}

Picture

Graph

Diagram

Map

Figure

All images must be numbered and mentioned in the text, should include the source image. The indications of the image: type and number of image, image title and source are written in 10 font size. In the text set as image maps, figures, graphs and charts-with the intention of not losing the formatting by the author.

\section{Equations and Formulae:}

When using equations or formulas should be used in Microsoft Word equation editor and numbered.

\section{Paper sending}

Entries must be sent to the email address: lgtz@uacj.mx. With Dr. Luis Enrique Gutiérrez Casas, editor of this publication.

Acceptance of each collaboration will depend on the evaluation of two examiners skilled in the art to be kept anonymous, like the author(s) for the same purposes. 
Publicación afiliada a la

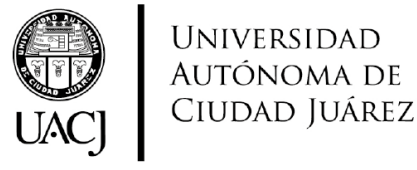

Red
RIED

Esta obra se editó y terminó de imprimir en Ciudad Juárez, Chihuahua, México 
